\title{
Linear and Nonlinear Dynamics of Micro and Nano- Resonators: Review of Recent Advances
}

\author{
A. Z. Hajjaj $j^{\ddagger}$, N. Jaber ${ }^{\ddagger, a, b}$, S. Ilyas ${ }^{\ddagger, a}$, F. K. Alfosail ${ }^{\ddagger}, \mathrm{a}$, and M. I. Younis ${ }^{\mathrm{a}, 1}$ \\ ${ }^{a}$ Physical Science and Engineering Division, King Abdullah University of Science and Technology, Thuwal, 23955-6900, \\ Saudi Arabia. \\ ${ }^{b}$ School of Mechanical Engineering, Purdue University, West Lafayette, IN, 47906,USA.
}

\begin{abstract}
Micro and nanoelectromechanical systems M/NEMS have been extensively investigated and exploited in the past few decades for various applications and for probing fundamental physical phenomena. Understanding the linear and nonlinear dynamical behaviors of the movable structures in these systems is crucial for their successful implementation in various novel technologies and to meet the long list of new sophisticated requirements. This paper presents a review for some of the recent topics pertaining to the dynamical behaviors, linear and nonlinear, of M/NEMS resonating structures. First, an overview is presented of the various used dynamical approaches to enhance the sensitivity of resonators for sensing applications. Then a summary is presented of the recent works on the linear and nonlinear mode coupling in M/NEMS resonator. Next, recent research is reviewed on coupled M/NEMS resonators, mechanically and electrically, leading to collective behaviors like mode localization. The final part of the paper discusses analytical approaches that have been developed to better understand and investigate the dynamical behavior of M/NEMS resonators focusing on the perturbation method the multiple time scales.
\end{abstract}

\section{Introduction}

Micro and nanoelectromechanical systems (MEMS and NEMS) have increasingly attracted considerable interest due to their small size, batch-fabrication, high reliability, and low power consumption. Therefore, M/NEMS were proposed in the past few decades for numerous potential applications including gas/mass sensing [1-3], filters [4-7] , memory devices [8-10], logic devices [11-13], and gyroscopes [14, 15].

To further exploit M/NEMS into various applications and to meet the ever increasing performance requirements, it is essential to understand the mechanical and dynamical behavior of the moveable structures of these devices; particularly those that are operated near their resonance frequencies (resonators). These resonators can have simple geometry, such as clamped-clamped bridged, cantilevers, and plates, or can be of more complex shapes. They can be

\footnotetext{
${ }^{\ddagger}$ A. Z. Hajjaj, N. Jaber, S. Ilyas, and F. K. Alfosail contributed equally to this work

${ }^{1}$ Corresponding author: Mohammad I. Younis, e-mail: Mohammad.Younis@kaust.edu.sa, ORCID: orcid.org/00000002-9491-1838
} 
driven into the linear or nonlinear regimes using different actuation mechanisms, such as electrothermal, piezoelectric, electrostatic, electromagnetic, and optical. In sensing applications, their dynamics are monitored by the change in frequency or amplitude resulted from the alteration of one or more control parameter(s), such as mass, applied force, viscous damping, surrounding gas/pressure, and temperature. M/NEMS resonators can be based on single (uncoupled) or coupled (mechanically or electrically) structures. Coupling can also occur with the various vibration modes of a single structure through phenomena, such as veering and internal (auto parametric) resonances. These structures can be excited at primary resonance, secondary resonances, at the fundamental mode, higher order modes, using a single harmonic source or multi-harmonic sources. Consequently, M/NEMS resonators can have rich and complex dynamics, which are not yet fully understood or exploited.

Recently, there have been considerable theoretical and experimental works that investigate the linear and nonlinear dynamics of M/NEMS and their exploitation for various applications; many of which were surveyed in [16-25]. M/NEMS have enabled in-depth studies of various fundamental phenomena thanks to the capability to precisely control different physical parameters, such as the boundary conditions, stiffness, damping, and geometry. Chaos [26], Hopf bifurcations [27], synchronization [28-30], internal resonances [31-33], and veering [34-36] are examples of physical phenomena that were extensively investigated at the micro and nano scale. Also, significant research has been conducted recently on nonlinear mechanics of microsystems, such as dynamic integrity of electrostatic MEMS [37-39] and AFM [40, 41], control of MEMS [42-44], reduced-order modeling and gyroscopes [45-47].

This work aims to present an in-depth review of selected recent topics related to the dynamics of M/NEMS resonators. The first part will focus on reviewing several types of dynamical approaches used to enhance the sensitivity of M/NEMS resonators for sensing applications; mainly higher order modes activation, bifurcation instabilities, and multimode excitation. The second part will focus on the linear and nonlinear modal coupling in M/NEMS and their exploitation in several potential applications, such as filters, synchronization, and frequency stabilization. The third part will review the M/NEMS coupled structures, mechanically and electrically, and focus on the mode localization phenomenon. The last part of this work will survey models based on the perturbation method, multiple scales technique, and discuss models to study the secondary resonances and nonlinear mode coupling (internal resonance) in M/NEMS structures. 


\section{Dynamic Approaches to Enhance Sensitivity of Resonators}

MEMS and NEMS resonators have been exploited for detecting ultra-minute particles in wide range of applications including antibiotic detections [48], vaccinia virus characterization [49], Xenon atom detection [50], and single cell imaging [51]. Also, they are used for selectively detecting ultra-low concentrations of particular gases, such as volatile organic compounds [52,53], nitro-toluene vapors, which is an example of explosive-related molecule [54], and carbon dioxide [55]. Selectivity towards specific pathogen or gas can be achieved by functionalizing the resonator surface with materials that have affinity toward specific molecules [56-58]. MEMS and NEMS resonators have been recently surveyed in several review papers that discuss some aspects of their performance, actuation and detection techniques, and potential applications [21, 22, 58-61]. Here we summarize several dynamic-based approaches to enhance the performance and functionality of resonators for sensing applications. These techniques provide methods to improve sensitivity and offer potential solutions to perform smart functionalities without the need for complex devices and circuits.

The operating principle of resonator-based mass sensors is based on monitoring the frequency shift $(\Delta f)$ in the resonance frequency $(f)$ due to the molecules adsorption $\left(\Delta m_{e f f}\right)$ on the resonator surface [62], Fig. 1. The responsivity of resonator $\Re$ is defined as

$$
\mathfrak{R}=\frac{\Delta f}{\Delta m_{e f f}}=\frac{-f}{2 m_{e f f}}
$$

where $m_{e f f}$ is the effective mass of the resonator, $f=\frac{1}{2 \pi} \sqrt{k_{e f f} / m_{e f f}}$ is the natural (resonance) frequency, and $k_{e f f}$ is the effective stiffness. 


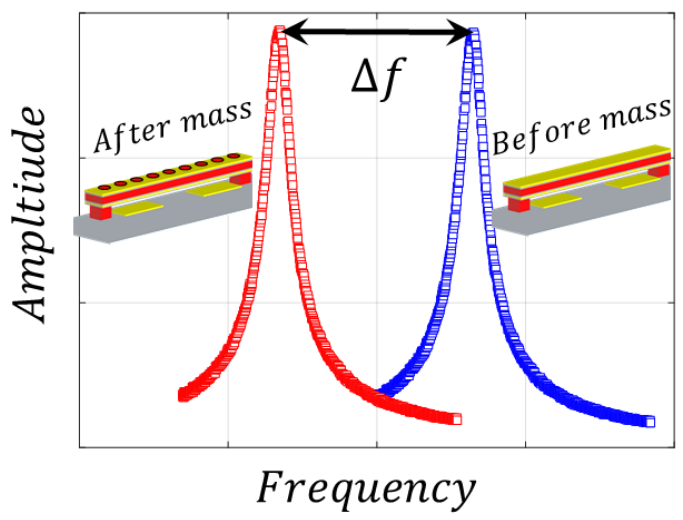

Fig. 1. The principle of operation of the frequency-shift-based resonant mass sensor.

Improving the resonator sensitivity has been the subject of intensive research in the last decade [63-68]. To achieve high sensitivity, most of the works have been toward shrinking the resonator size, for example from the micro to the nanoscale, which increases the resonator frequency and decreases the device mass. This leads to highly responsive mass sensor as can be inferred from Eq. (1). By utilizing different resonators geometries and materials, mass sensors with wide range of sensitivities have been reported at the nanogram $\left(1 \mathrm{ng}=10^{-9} \mathrm{~g}\right)$ [69], picogram $\left(1 \mathrm{pg}=10^{-12} \mathrm{~g}\right)$ [70], femtogram $\left(1 \mathrm{fg}=10^{-15} \mathrm{~g}\right)$ [71, 72], attogram $\left(1 \mathrm{ag}=10^{-18} \mathrm{~g}\right)$ [73], zeptogram $\left(1 \mathrm{zg}=10^{-21} \mathrm{~g}\right)$ [74], and yoctogram $\left(1 \mathrm{yg}=10^{-24} \mathrm{~g}\right)$ [50]. However, shrinking the size increases the time required to perform mass spectrometry analysis due to the small surface area of the device [75] and reduces the surface area available for functionalization [67]. Also, nanoresonators require complex measurement techniques and controlled testing conditions to detect the vibration motion, which hinder the practicality of these sensors. Lochon et al. [67] compared between shrinking the resonator size and the higher order mode excitation in improving the resonator sensitivity. They experimentally proved that using the higher order vibration modes and shrinking size when operating at the fundamental mode have the same effect on sensitivity improvement. Hence, using the higher order modes preserves the surface area available for coating and improves the functionalization reproducibility. Also, utilizing the higher order modes increases the quality factor, thereby leading to a high signal-to-noise ratio, which is essential for ultra-sensitive sensors $[66,76]$. However, exciting the higher order modes is challenging and requires large actuation force that may not be available using conventional power generators. Several researchers proposed different techniques to enhance the excitation of higher order modes using low voltages. Jin et al. [66] proposed and developed an electromagnetic excitation method that can efficiently 
excite the second mode of vibration. The method is based on applying the force at the maximum deflection points of the second mode shape. They showed six times improvement in the sensor responsivity.

In electrostatically actuated resonators, different electrode configurations have been proposed to enhance the excitation of the higher order modes, at which the electrodes are designed to match the shape of the excited mode. Using this technique, Okada et al. [77] increased the amplitude of the third mode to be comparable to the first mode using the same power level. Similarly, Kuroda et al. [78] improved the excitation of the first five bending modes of a fishbone-shaped clamped-clamped microbeam with a high rejection ratio. Jaber et al. [79] experimentally and analytically studied the effect of the lower electrode design on the excitation of higher order modes of clampedclamped microbeams as compared to the first mode, Fig. 2a. They demonstrated effective excitation of the second mode using a half electrode configuration, Fig. 2b. Also, by using a two-third lower electrode configuration, Fig. 2c, they reduced the voltage required to activate the third mode to one third compared with the full electrode configuration. Lakshmanan and Mutharasan [80] studied the effect of the resonator dimensions on the higher order modes excitation. They showed that higher order modes have strong dependence on the width of the microbeam where reducing the width amplifies the amplitude of vibration.

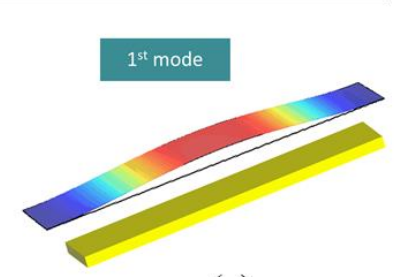

(a)
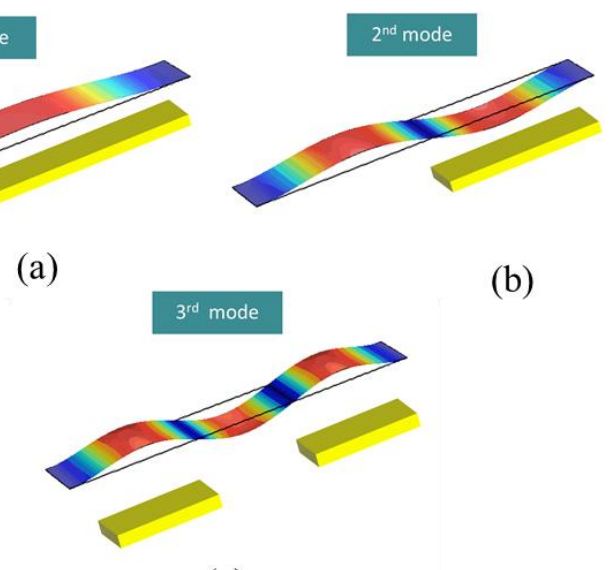

(c)

Fig. 2. Clamped-clamped mode shapes with different lower electrode configurations. (a) Full electrode. (b) Half electrode. (c) Twothird electrode.

Another approach to improve the sensitivity is by exploiting the pull-in instabilities of electrostatically actuated resonators. The pull-in instability was first reported in 1967 [81]. Since then several studies have been focused on understanding this phenomenon using different analytical approaches and finite element methods [59, 82-85]. Pull-in 
can be triggered when the electrostatic force acting on the movable electrode overcomes its restoring elastic force resulting into its collapse on the stationary electrode. The pull-in can be classified into static pull-in due to the large DC bias, and dynamic pull-in, which can be activated when the microbeam is dynamically actuated near the resonance frequency [84]. The collapse of the movable electrode into the stationary one is commonly considered undesirable and might lead to the damage of the MEMS structure, and hence needs to be controlled and predicted [59, 84-87]. Conversely, several devices have employed this phenomenon in different applications, such as earthquake detection [88], demonstrating ultra-sensitive accelerometer [89], extracting the mechanical properties of MEMS structures [79], and also in enhancing the sensitivity of mass and gas sensors [90-93]. Khater et al. [90] demonstrated a lowconcentration ethanol sensor by operating a microcantilever near the static pull-in instability. They experimentally detected 5 ppm of ethanol vapor. Jaber et al. [91] utilized the dynamic pull-in instability, generated from sweeping large AC harmonic excitation around resonance, to demonstrate a smart sensor that can measure the gas concentration and autonomously perform switching without the need of microcontrollers and complex circuits. As shown in Fig. 3, the idea is based on operating the resonator at a fixed frequency near the dynamic pull-in frequency band (where a resonator cannot sustain stable oscillation). As the gas is adsorbed on the resonator surface, the total mass of the sensor increases, which shifts the frequency response curve to the left. Once the amount of adsorbed mass exceeds a threshold value, the operating frequency falls inside the pull-in band, which causes the upper electrode to collapse into the lower electrode. By designing an appropriate read-out circuit, this can be utilized to activate an action, for example an alarm. Since higher order modes are utilized to enhance the sensitivity of resonator-based mass sensors, actuating the resonator near the pull-in band of the higher order mode should lead to more sensitive sensors. However, realizing a pull-in band near the higher modes requires large actuation voltages that might not be available using conventional power generators [79]. Jaber et al. [94] combined the pull-in instability near the first mode, which needs relatively low voltages to be activated, and the high sensitivity near the third mode to demonstrate an ultra-sensitive smart switch. 


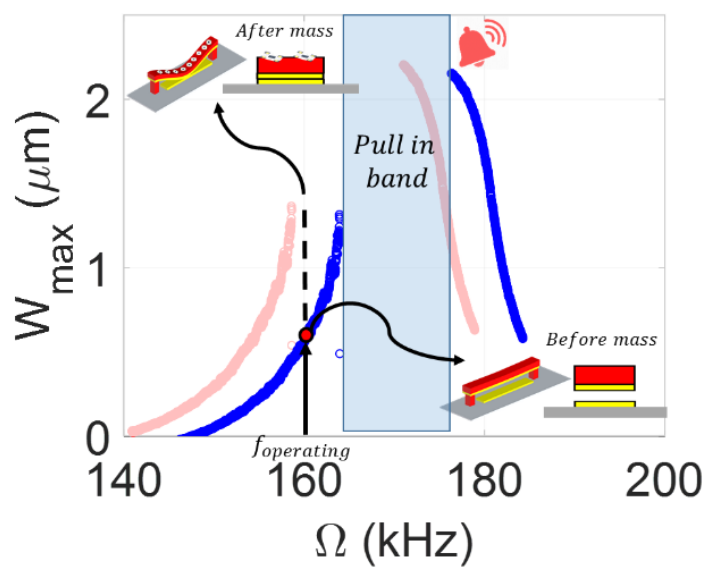

Fig. 3. The operating principle of a smart sensor based on the dynamic pull-in instability. The vertical arrow shows the operating frequency, which is initially outside the pull-in band (blue curve). The difference between the operating frequency and the pull-in band defines the threshold value. Once the amount of mass exceeds the threshold value, the operating point falls inside the pull-in band (red curve) and the upper electrode collapses into the lower electrode, which can be employed to activate an alarm.

Detecting bifurcation points has been explored to enhance the sensitivity of resonators for gas and mass sensing applications $[85,91,92,95-97]$. The main principle is based on exploiting the sudden jump in amplitude by operating the resonator near the bifurcation points. As shown in Fig. 4, operating the resonator at point A' amplifies the sensor sensitivity to a physical stimuli change at which there is continuous increase in amplitude as the response shifts from A' to the bifurcation point A leading then to a sudden amplitude jump, as shown in the inset of Fig. 4 [97]. Nguyen et al. [95] proposed a sensing technique based on the bifurcation and multistability in a hysteretic frequency response of an electrostatically actuated MEMS resonator. Analytically they demonstrated the possibility of detecting very small mass by tracking the sudden jump in amplitude near the bifurcation point. In [98, 99], a two order of magnitude sensitivity improvement is demonstrated by utilizing the sudden jump in amplitude due to the activation of parametric resonance in comparison to the simple harmonic resonance. Younis and Alsaleem [100] exploited different bifurcations and the dynamic pull-in band near primary and secondary resonances to demonstrate new concepts for mass sensing using MEMS resonators. They experimentally demonstrated that the sudden jump in the amplitude near the subharmonic resonance produces the most promising results. Kumar et al. [85] demonstrated analytically and experimentally a mass sensor based on the amplitude jump near cyclic-fold bifurcations. Cho et al. [64] exploited the 
cubic nonlinearity of a carbon nanotube resonator to increase the resonance frequency up to three times the linear natural frequency reaching femtogram sensitivity. However, the use of nonlinear instabilities for sensing rely on the robustness of the operating point. As the operating point approaches the bifurcation point, the basin of attraction gets eroded, which may lead to noise induced jumps or switching and reduce the reliability of the sensor readings. Hence, the stability of the operating point needs to be ensured to avoid erroneous measurements, which can be studied by conducting global dynamic analysis [37-39].

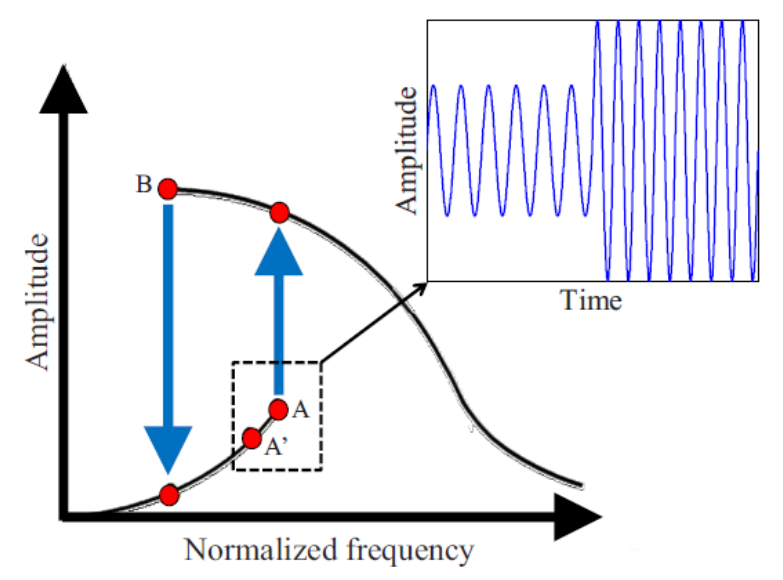

Fig. 4. The operating principle of the bifurcation-based sensor at which point A and B lie on the saddle-node bifurcations. As the frequency response shifts due to the physical stimuli change, the amplitude experiences a rapid jump that can be easily measured, which allows the detection of ultra-small masses. Inset: shows the rapid change in amplitude as the operating points move across the bifurcation point. Reprinted with permission from [97].

Recently, simultaneously monitoring the frequency shift of multiple modes of vibration has gained significant attention for wide range of applications, such as sensing [63, 66-68, 80, 101-103], signal filtering [35], and Atomic force microscopy (AFM) [104-106]. Analytically and experimentally, Dohn et al. [65] proved the ability to extract the position and mass of a gold particle on the surface of a microcantilever resonator by measuring the frequency shift of the first four modes while changing the location of the gold particle. The results were in good agreement with the visual inspection of the gold particle position. Using a perturbation analysis, Bouchalaa et al. [107] developed a closed form formula to determine the position and the mass of a particle adsorbed on a clamped-clamped microbeam surface. The analytical results were in good agreement with the experimental measurements. By tracking two modes of 
vibration, Hanay et al. [3] demonstrated the possibility of realizing the position of adsorbed antibodies on the resonator surface in real time. As shown in Fig. 5, by tracking the frequency shift of the first four modes of vibration of a microcantilever, Olcum et al. [68] monitored the position, velocity and mass of nanoparticles flowing inside a microchannel. Figure $5 b$ shows the effect of the particle position on the resonant frequency of the first four modes of vibration. Figure $5 \mathrm{c}$ shows the measured frequency due to two nanoparticles of radius 150-nm and 100-nm flowing inside the microchannel, which clearly shows the effect of the particle mass and position on the measured frequency shift of the first four modes. The 100-nm one induces a smaller frequency shift due to the lower mass compared with the 150-nm particle. Also, it demonstrates that as the particle passes the microcantilever tip, the measured frequency shift is maximum, whereas when it crosses the nodal points the frequency shift is zero. Also, they developed a platform that can record the fast transient signal, which enabled the tracking of multiple nanoparticles flowing simultaneously. This result is important in analyzing the mass distribution of analytes inside a flowing solution. By employing the multimode of a micromechanical bulk resonator, the phase transition of polyethylene Glycol from solid to liquid is qualitatively analyzed, and the results were verified optically. By monitoring the frequency shift in the first and second modes of vibration, Jaber et al. [1] demonstrated a smart sensor that can simultaneously measure the environmental temperature and humidity using a single MEMS resonator, which reduces the overall sensor footprint, the power consumption, and the fabrication cost. Younis [108] studied the dynamics of a clamped-clamped microbeam simultaneously actuated near the first and third modes of vibration. The complex dynamics of the system has been highlighted through phase diagrams, Poincare-sections, bifurcation diagrams, and FFTs showing the quasi-periodic bifurcation route to chaos. The multimodal excitation and detection of an AFM cantilever has been used to generate information about the surface topography and properties of materials. In this technique, each mode acts as a different channel of information sensitive to specific material characteristic (topography, external forces, damping, and Young's modulus). The multimodal feedback modulation is operated in different feedback configurations, either frequency feedback modulation, amplitude feedback modulation, or both [109]. Analytically, Chawla et al. [104] compared between the different configurations in extracting the sample characteristics. They concluded that the constant amplitude phase locked loop explores a wide range of interactions [104]. By simultaneously exciting and detecting the first two eigenmodes of an AFM cantilever, the intermolecular structure of antibodies is identified in [110] without destroying the noncovalent bonds. Rodrigues et al. [111] excited the first and second mode of the AFM cantilever to extract the image of the sample and the compositional change. 
(a)

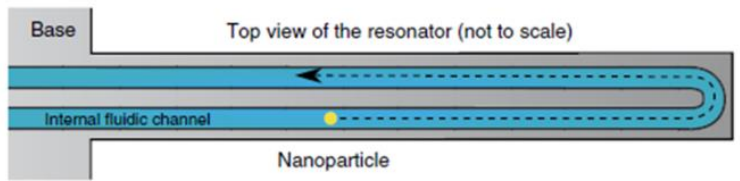

(b)

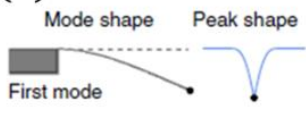

(c)
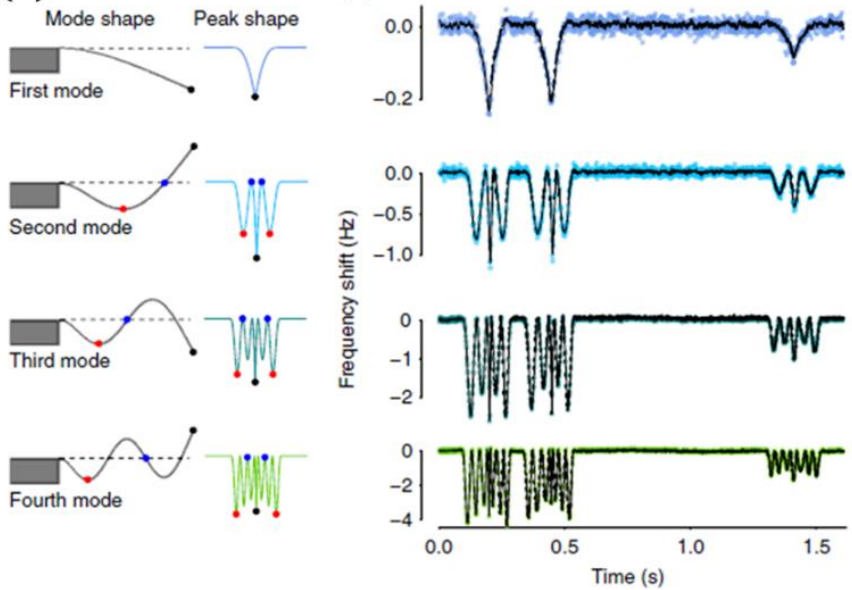

Fig. 5. Measuring the mass and position of particles flowing inside a microchannel. (a) Schematic of the microcantilever with the embedded microchannel showing the nanoparticle. (b) The first four modes of vibration and the corresponding frequency shift at different points along the microcantilever length. (c) The measured frequency shift near the first four modes of vibration as two 150-nm particles and one 100-nm particle flowing inside the microchannel. Reprinted with permission from [68].

As can be inferred from this section, several dynamical concepts have been investigated to improve the sensitivity of resonator-based sensors and to utilize a single device to measure multiple physical parameters. Although the reported findings are promising, the practical implementation is still facing several challenges and requires extensive research. Monitoring the change in the bifurcation point is challenging requiring complex control systems to suppress the noise effect and to measure the changes in the physical stimuli. Also, the simultaneous excitation and detection of multiple modes need complex read-out circuits to accurately record the change at each mode of vibration. Such challenges call for further research dedicated to the exploitation of exciting dynamical phenomena for resonant sensing.

\section{Linear and Nonlinear Modal Coupling}

In the past two decades, mode coupling among the various vibration modes has been increasingly investigated in micro and nanostructures. This was motivated to achieve better understanding of complex fundamental dynamical 
phenomena or for developing new applications. The coupling of those modes can be either mechanically [26, 112$115]$ or electrically $[28,30,116]$. Also, they can be coupled linearly or nonlinearly among the structure itself $[35,117$ 120], for instance through internal resonances [32, 121, 122].

Several groups $[32,123-128]$ have developed mathematical models to explain and explore the energy transfer among vibration modes using lumped-mass parameter systems or distributed parameter models using for instance the EulerBernoulli beam equation [32, 123]. For example, Zanette [125-127] performed in-depth theoretical analysis on the modal coupling and energy transfer among vibration modes of micro and nanomechanical systems.

Motivated by the theoretical analysis of the mode coupling of M/NEMS, several groups demonstrated and explored experimentally the modal coupling of mechanical structures and proposed them for potential applications. They explained the energy dissipation and nonlinearity control of different modes using the modal coupling between different modes [26, 27, 29, 30, 112-116, 119, 124, 129-139]. One of the earliest works on NEMS resonators was the work of Karabalin et al. [26] who demonstrated a complex nonlinear dynamic behavior based on two elastically coupled NEMS resonators, Figs. 6a. The two coupled resonators were driven independently. Period doubling transition, as well as chaotic behaviors, were demonstrated. Mahboob et al. [27] showed that the simultaneous activation of hybrid modes in a coupled microresonator can lead to the reduction or elimination of nonlinear dissipation, Figs. 6b. The authors demonstrated that as passing through Hopf bifurcation, period doubling, and multistable response, the system undergoes a self-sustained oscillation that could be useful for different novel applications. Modal coupling was also used to detect higher order modes as driving a bridge resonator around its fundamental frequency. Westra et al. [134] investigated experimentally and theoretically the modal coupling between different flexural modes of a clamped-clamped microbeam to be used as a self-detector of the amplitude for other modes, Fig. 6c. The authors proposed a technique to measure the stored energy in a specific resonant mode. The same group [140, 141] demonstrated that the strong coupling between the flexural modes of a clamped-clamped microbeam enables the measurement of the frequency response of the targeted mode via amplitude-phase modulation. The fundamental mode was used as a mechanical detector for the weakly driven resonator. 

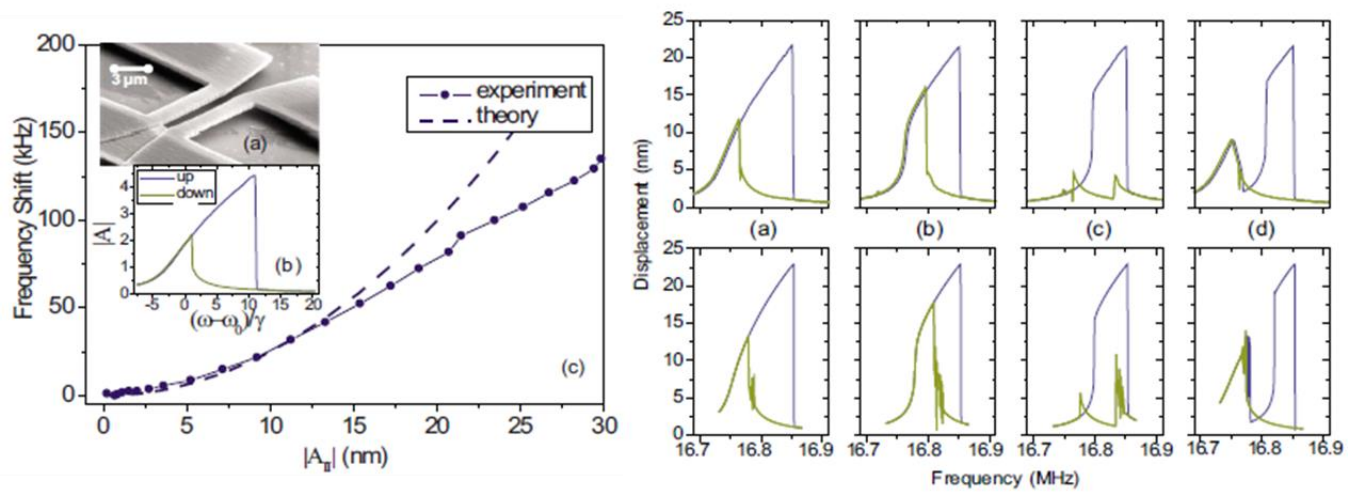

(a)
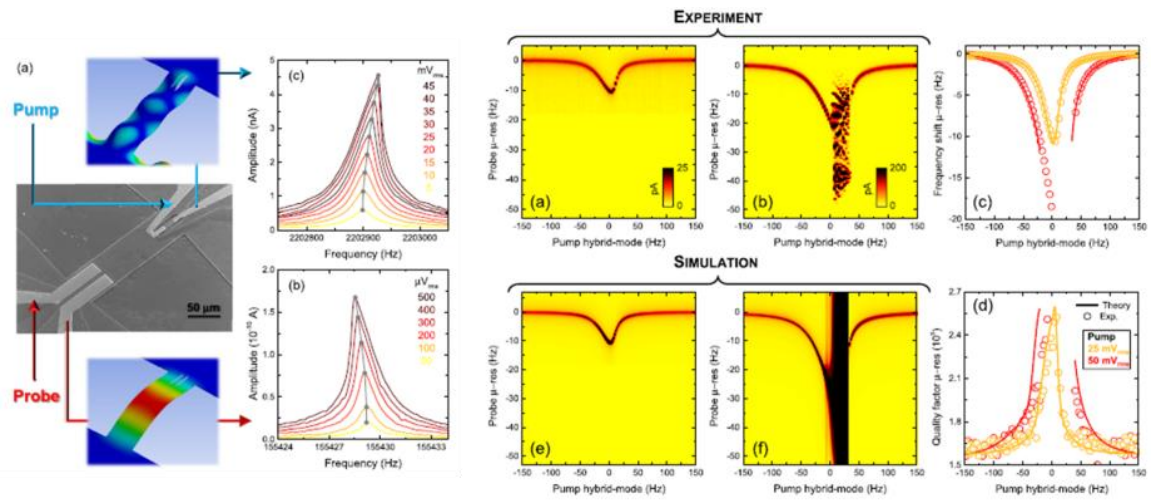

(b)
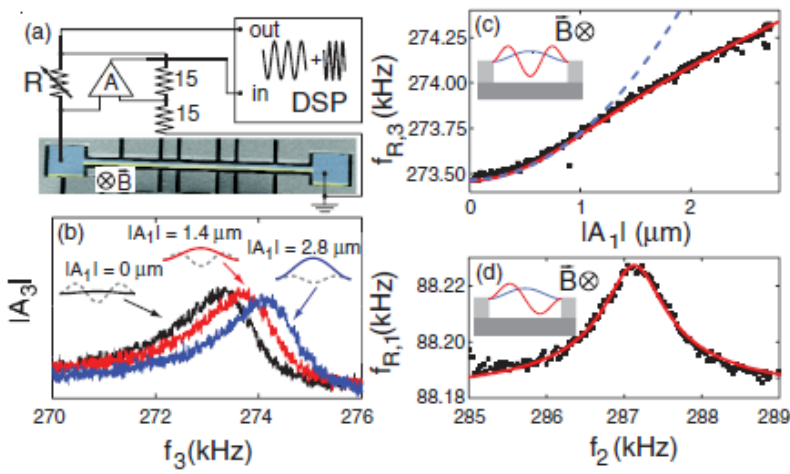

(c)

Fig.6: (a) Modal interaction in two coupled nanomechanical resonators [26]. The figure displays the first mode frequency shift of the coupled nanomechanical resonator that is shown to be dependent on the vibration amplitude of the second mode. The left-hand side of the figure shows the frequency response of fundamental mode for stronger driving of the first mode. (b) Hopf bifurcation of a coupled microresonator [27]. The coupled electromechanical system consists of a nanomechanical resonator fixed into the clamping point of a micromechanical resonator. The microresonator frequency response shows the Hopf instability. (c) The modal 
interaction of a clamped-clamped microbeam [140] showing the detection of the higher order mode using the resonance frequency shift around the fundamental frequency. Reprinted with permissions from [26, 27, 140].

In addition to silicon-based structures, modal coupling was also studied in 2D circular graphene membranes [142, 143] and carbon nanotubes (CNTs) [144-146]. Further, modal coupling was demonstrated in systems with highly tunable stiffness $[34,35]$, in which the natural frequencies are adjusted to certain ratios leading to strong coupling among different modes of vibration. This coupling can be linear, via the veering phenomenon for example, or nonlinear via internal (autoparametric) resonance.

The veering phenomenon has long attracted several researchers in the classical structural dynamics, mainly in cablespring systems [147, 148], plates [149, 150], and curved beams [151, 152]. Veering (avoided-crossing) occurs when two frequencies approach each other as varying a control parameter due to a linear coupling between two modes and then deviate away from each other, and each one continues along the path that the other would have taken if they would cross. Henceforth, both modes get affected, in the veering zone, by the shape of each other, known as mode hybridization. In M/NEMS, veering was studied mostly when associating with the mode localization phenomenon occurring in weakly coupled systems $[135,153]$. Mode localization will be reviewed in-depth in the following section. Veering was also explored for internally coupled modes in resonators with tunable stiffness. One method to tune stiffness is through the electrothermal actuation, Joule's heating effect, which was used recently to highly tune and control the resonance frequencies of M/NEMS resonators [35]. In recent works, Hajjaj et al. [34,35] showed that the veering phenomenon among the first two symmetric modes of an initially curved (arch) MEMS resonator electrothermally tuned can be strongly activated. The first resonance frequency of the arch beam is shown to increase up to twice its fundamental value while the third resonance frequency is shown to decrease until getting very close to the first resonance frequency triggering the veering phenomenon, Fig. 7a. An exploitation of the nonlinear softening, hardening and veering phenomena (near crossing) of the arch beam was reported to realize a band-pass filter. By electrostatically driving both modes nonlinearly near the veering regime, such that the first (dominated with the quadratic nonlinearity) and third (dominated with the cubic nonlinearity) modes exhibit softening and hardening behavior, respectively, a sharp roll off from the passband to the stopband is achieved, Figs. 7b-d. Hajjaj et al. [34, 35] demonstrated also experimentally and theoretically the exchange of nonlinearities between both modes as passing the veering zone. In addition to microbeams, the veering phenomenon was investigated for CNTs [36, 154, 155]. Several 
studies $[36,155]$ investigated veering on clamped-clamped slightly curved (slacked) CNTs. Notably, these slacked CNTs showed a potential transfer of energy from the first targeted mode to up to the ninth (untargeted) mode [155].
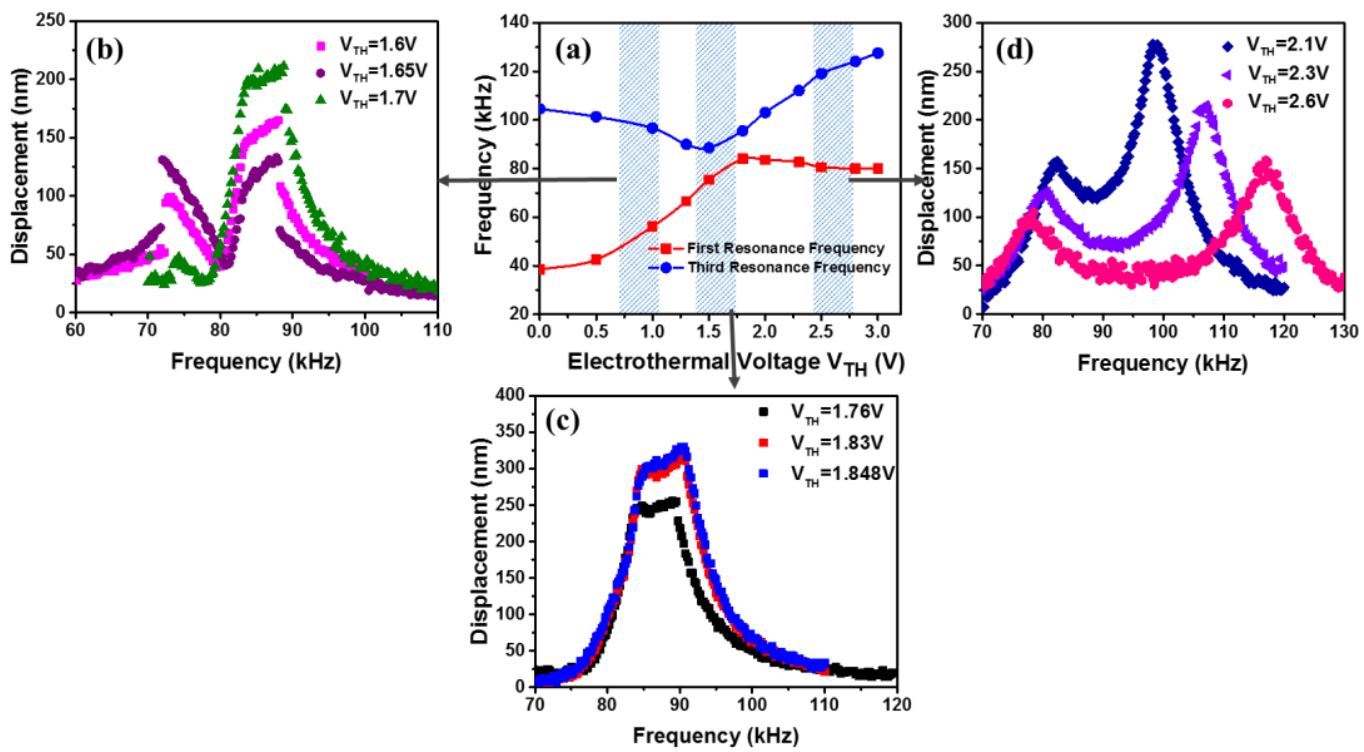

Fig. 7: Veering phenomenon of an electrothermally tuned and electrostatically actuated arch resonator [35]. (a) The variation of the two symmetric resonance frequencies of the arch beam with the applied electrothermal voltage. (b)-(d) The frequency response curves of the arch beam before (b), on (c), and after (d) veering demonstrating the realization of a flat band-pass filter as operating the arch beam around veering (c). Reprinted with permission from [35].

\subsection{Internal Resonance}

One primary mechanism of nonlinear coupling between different modes in structural mechanics is internal resonance. It represents a mechanism for energy transfer from the targeted vibration mode to another mode either in the same $[32,33,123,156]$ or different plane of vibration $[31,157,158]$. One necessary condition to activate internal resonance is to have an integer ratio between the involved modes. Typically, the contributed mode represents a significant source of energy or a sink of energy storage that is targeted for detection and measurements. One-to-one [122, 123, 159], two-to-one $[32,33,156,157,160,161]$, and three-to-one $[30,31,33,123,156]$ internal resonances are the most investigated in the literature. A recent work on four-to-one internal resonance has been also reported [33].

In the past few decades, internal resonance in M/NEMS has been widely studied in various structures including cantilevers $[162,163]$, arch beams[32, 123, 156], membranes from 2D material (such as graphene and $\mathrm{MoS}_{2}$ ) [122, 142], CNTs [164], and electrically and mechanically coupled structures [31, 158, 161]. 
The interest to study internal resonance in M/NEMS resonators has been boosted to achieve better fundamental understanding of the phenomenon and for using it in potential applications, such as energy harvesting [165], frequency stabilization [31, 33], and synchronization [30]. Until almost a decade ago, the investigation of internal resonance was mostly theoretical where several groups $[123,160,166,167]$ examined the nonlinear behavior of different structures as experiencing internal resonance using analytical methods, such as the multiple scales method.

One of the earliest experimental work was that of Antonio et al.[31] who showed that the oscillation frequency stability of a nonlinear self-sustained MEMS resonator could be improved by operating the resonator in the internal resonance regime. As experiencing the nonlinear coupling through internal resonance, the contributed mode absorbs the frequency and amplitude fluctuation of the targeted mode, Fig. 8. The same group [158] has recently shown a novel technique to support a self-sustained oscillation, via internal resonance, to compensate for energy losses. They exploit internal resonance as a way to store the mechanical energy among different vibrational modes and transfer it back to the targeted mode when the external excitation is switched off.

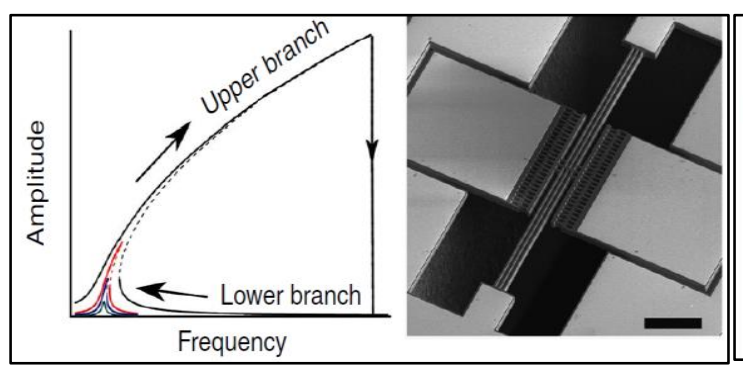

(a)

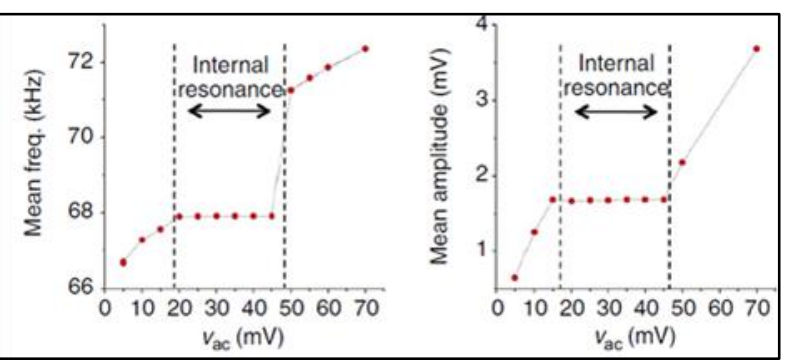

(b)

Fig. 8. Frequency stabilization via internal resonance [31]. (a) The clamped-clamped resonator and the corresponding frequency response curve around its fundamental frequency in an open loop configuration. (b) The mean frequency and amplitude of the fundamental mode with the excitation voltage as experiencing internal resonance. Reprinted with permission from [31].

On the other hand, one of the simplest known structures to exhibit different types of internal resonance are initially curved MEMS resonators. Recently, these structures have been proposed for several potential applications, such as filters [35], sensors [168], and logic/memory gates [11]. Arch MEMS resonators are known for their rich dynamical behavior [169, 170] including bistability, snap-through motion, and more importantly inherent nonlinearities; quadratic, from initial curvature, and cubic, from stretching. More interestingly, the natural frequencies of the arch beam are shown to be sensitive to axial loads [34], which enables the realization of several commensurate ratios among various modes $(1: 1,2: 1,3: 1$, and 4:1). This leads to the possibility of activating different types of internal resonances 
using these simple structures. Younis and coauthors $[32,33,123,156]$ studied profoundly different types of internal resonances of arch resonator theoretically $[32,123]$ and experimentally $[32,33,156]$.

Ramini et al. [156] investigated experimentally, using optical techniques, the 2:1 and 3:1 internal resonances of an arch beam electrothermally tuned and electrostatically actuated. Both internal resonances occurred between the first two symmetric modes of vibration. Classical frequency response curves of internal resonance (M-shaped curves) were reported experimentally showing the nonlinear interaction between the contributing modes. In the same context, same group [32] investigated the effect of direct excitation of the second symmetric resonance frequency as experiencing 2:1 internal resonance while exciting the first vibrational mode. Indeed, the operation of the arch beam at the ambient pressure leads to simultaneous excitation of the third vibrational mode as experiencing the 2:1 internal resonance. A complex behavior was reported experimentally and theoretically, where the arch passes through unstable branches leading to quasi-periodic motion, Hopf bifurcation and chaotic behavior. More recently, Hajjaj et al. [33] investigated using electrical techniques the activation of different internal resonances $(2: 1,3: 1$, and $4: 1)$ between the first two symmetric modes of an arch beam as tuning its stiffness electrothermally and driving it electrostatically, Fig. 9. The storing and redistribution of the energy of both contributing modes were demonstrated and quantified for each type of internal resonance. As driving the system highly, the arch beam was shown to undergo more complex dynamical behavior, as experiencing internal resonance, leading the system to pass by an unstable motion that leads to Hopf bifurcation and chaotic behavior. An improvement of the frequency stabilization was reported as driving the arch resonator in the internal resonance regime as shown previously by Antoine et al. [31]. Internal resonance was also observed and exploited in carefully designed structures to get commensurate ratios between different modes such as the H-shapes structures [161] and the microcantilevers with outer and inner paddles [162, 163].

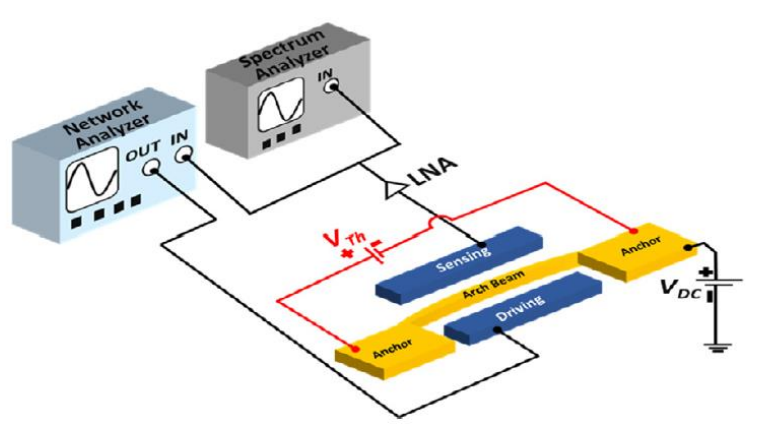

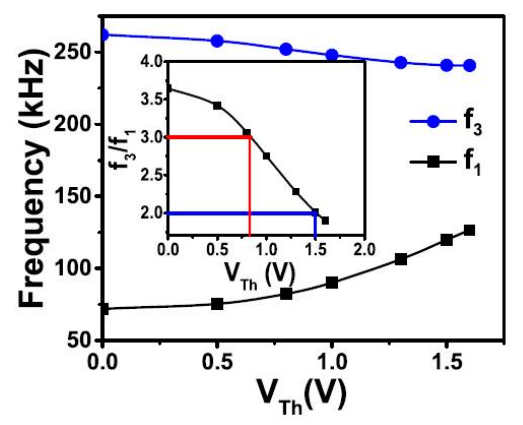

(a) 


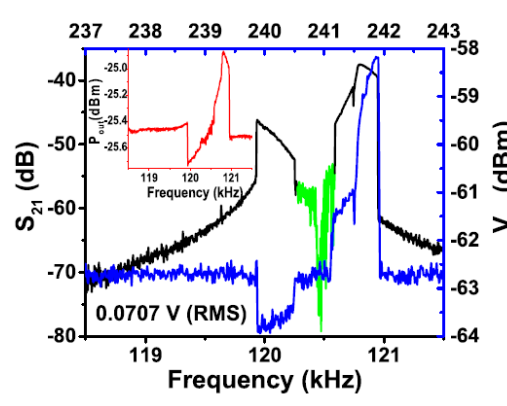

(b)

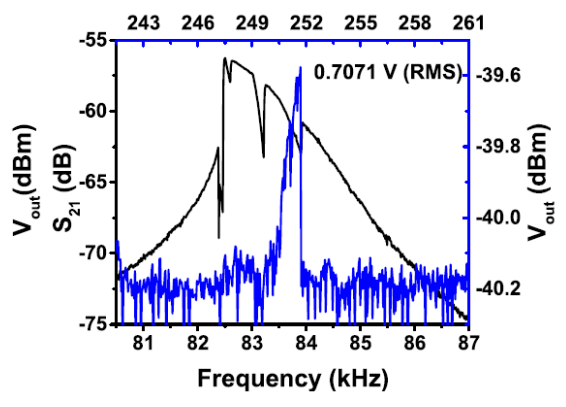

(c)

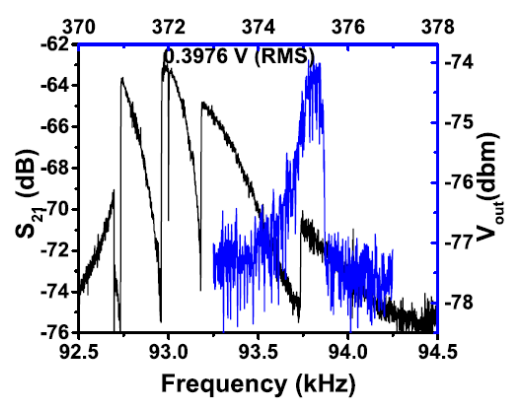

(d)

Fig.9. Multiple internal resonances of an electrothermally tuned and electrostatically driven arch MEMS resonator [33]. (a) The experimental setup and the variation of the resonance frequencies with the applied electrothermal voltage. (b)-(d) The frequency curves and the contribution of different modes as experiencing the 2:1 internal resonance, (b), 3:1 internal resonance, (c), and 4:1 internal resonance, (d). Reprinted with permission from [33].

As conferred in this section, mode coupling among different modes of vibrations has been investigated deeply in the literature using M/NEMS resonators. Basically, the investigation of mode coupling phenomenon was done to explore different associated physical phenomena (chaos, Hopf bifurcation, internal resonance, etc...) or to avoid them as implementing M/NEMS resonators in potential applications. Although the fundamental understanding of several linear (veering, crossover) and nonlinear mode coupling (internal resonance) was demonstrated, the integration of these phenomena in several potential applications, such as sensing, communication, and energy harvesting, is still limited and needs further investigation. For instance, more research is needed to control the energy transfer between different modes as being coupled linearly or nonlinearly. The control of energy transfer might be done for instance by controlling the pumped energy into the system or via other active control techniques. Also, accurate monitoring of the coupling (mechanical, electrical or in the structure by itself) will lead to better monitoring of the energy transfer among the various involved modes.

\section{MEMS/NEMS Coupled Resonators}

The dynamics of MEMS resonators become more interesting and more complex as these resonators are coupled among each other. Hence, investigating coupled M/NEMS resonators both theoretically and experimentally has been an active area of research particularly for applications in sensing and communication. Various types of resonators can be 
coupled together, electrostatically, mechanically, or both. Mechanically coupled structures have a physical element attached to the coupled resonators, while electrostatic coupling is due to a potential difference between the coupled resonators placed very close to each other.

\subsection{Modeling}

In this section, we discuss the modeling of two coupled resonators. A simple model for two mechanically coupled systems can be represented using lumped parameter spring-mass-damper systems. The equations of motion in this case, Fig. 10(a), are given by

$m_{1} \ddot{x}_{1}+c_{1} \dot{x}_{1}+\left(k_{1}+\delta k\right) x_{1}-\delta k x_{2}=F \cos (\Omega t)$

$m_{2} \ddot{x}_{2}+c_{2} \dot{x}_{2}+\left(k_{2}+\delta k\right) x_{2}-\delta k x_{1}=0$

where $m_{i}$ is the mass, $c_{i}$ is the damping coefficient, $k_{i}$ is the stiffness, $x_{i}$ is the displacement of $i^{\text {th }}$ resonator $(i=1,2)$,

$t$ is time, $\delta k$ is the coupling coefficient, and $F$ is the harmonic load of an excitation frequency $\Omega . \delta k$ is an important parameter that governs the coupling strength. In the case of mechanical coupling, $\delta k$ is commonly controlled by the location of the coupling element. The coupling is stronger if the coupler is attached to the maximum velocity points of the resonators, while the coupling is weaker when attached to low velocity points [171]. In addition to location, the coupling strength can be controlled by the material and dimensions of the coupler. However, once fabricated the strength of the coupling cannot be tuned significantly.

A distributed parameter model has also been used for two mechanically coupled clamped-clamped microbeams [172].

The eigenvalue problem of the system is solved by dividing the coupled system into five elements and solving for each of these elements using a total of 20 boundary conditions. The perturbation method of multiple time scales has been used to investigate the dynamic characteristics of the system for a MEMS filter application. A distributed model becomes necessary when dealing with the dynamics of higher order modes of the coupled system. However, such models can be complex and computationally expensive especially for more than two coupled structures.

Electrostatically coupled structures do not have a physical coupling element but rely on the potential difference between the two or more closely placed resonators. Figure 10(b) shows a spring-mass-damper system representing two electrostatically coupled resonators. The equation of motions for such system can be written as 
$M_{1} \ddot{X}_{1}+C_{1} \dot{X}_{1}+K_{1} X_{1}=F \cos (\Omega t)+\frac{\varepsilon A V_{D C 2}^{2}}{2\left(D-X_{1}+X_{2}\right)^{2}}$

$M_{2} \ddot{X}_{2}+C_{2} \dot{X}_{2}+K_{2} X_{2}=-\frac{\varepsilon A V_{D C 2}^{2}}{2\left(D-X_{1}+X_{2}\right)^{2}}$

where $M_{i}$ is mass, $C_{i}$ is the damping coefficient, $K_{i}$ is the stiffness, $X_{i}$ is the displacement of the $i^{\text {th }}$ resonator $(i=1,2), \varepsilon$ is the permittivity of the medium, $A$ is the electrostatic overlap area, $D$ is the operating gap, $t$ is time, $V_{D C 2}$ is the DC polarization voltage, and $F$ is the harmonic load of an excitation frequency $\Omega$. The advantage of using electrostatic coupling is the large tunability of the coupling strength, which is particularly useful in filter applications for tunable bandwidth [173-176]. The study in [177] is for electrostatically coupled cantilever microbeams, where the static, eigenvalue, and dynamic problems are solved and compared with experimental data. One conclusion of this investigation is the reduction in pull-in voltage $(\sim 36 \%)$ and pull-in time $(\sim 38 \%)$ of a coupled beam configuration when compared with a single beam.

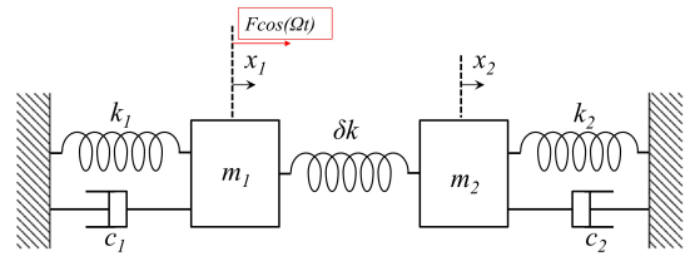

(a)

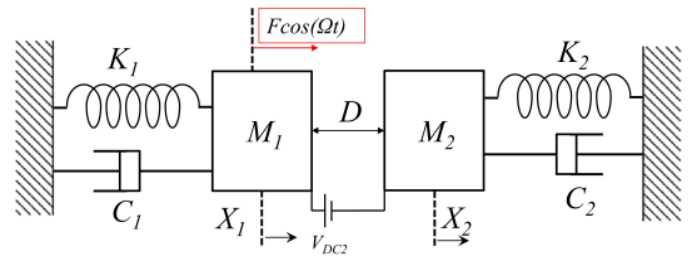

(b)

Fig. 10. Schematic of two (a) mechanically, (b) electrostatically coupled resonators represented by spring mass damper systems.

Resonators can also be modeled in the electrical domain using the electrical equivalent elements of stiffness, damping, and mass in capacitance, resistance, and inductance, respectively. The RLC circuit model representation of the resonator in an electrical domain has been extensively used for RF communication applications, where it helps in design optimization and evaluating various performance parameters [173-181]. Both electrostatically and mechanically coupled resonators can be modeled using this technique.

\subsection{Applications of Coupled Resonators}

M/NEMS coupled resonators have been extensively used for applications in filters, mixers, and amplifiers. In fact, a filter application based on coupled microbeams resonators was discussed very early in the MEMS technology in the 
seminal work in [81]. Another leading work in this area utilizes mechanically coupled and electrostatically actuated clamped-clamped microbeams [178]. This work presents a lumped parameter and an equivalent electrical circuit model. Detailed design criteria for ensuring a high performance filter is developed and validated with experiments. It is recommended that for the filter application, the mechanical coupler should be attached at quarter wavelength from the anchor point to ensure high quality factor device [182]. Quarter-wavelength coupling alleviates the mass loading effects caused by similar dimensions of the beam and coupler. A similar structure was then also proposed as a simultaneous mixer and filter, "mixler" [180].

Different coupled structures have been exploited for enhancing performance parameters of a filter including quality factor [178, 183-185], operating frequency [178, 186], and bandwidth tunability [173, 174, 187]. Piezoelectric coupled resonators have also been successfully employed to develop high performance filters [188-190]. Single chip multifrequency rectangular and ring-shaped aluminum nitride contour mode resonators were proposed for filtering [191]. The study in [191] showed an electrical cascade of devices to develop low insertion loss (4 dB at $93 \mathrm{MHz}$ ), and large rejection (27dB at $236 \mathrm{MHz})$. Both electrical and mechanical coupling have also been used together to enhance the filter performance [192]. In [192] two electrostatically coupled filters were studied, each comprising of two mechanically coupled clamped-clamped beam resonators. A dual bandpass filter centered around $27.5 \mathrm{MHz}$ and 27.8 MHz with a bandwidth of $0.6 \%$ and a $22 \mathrm{~dB}$ inner stopband rejection was achieved. The principles of internal mechanical and electrical phase inversion of coupled resonator arrays have been demonstrated experimentally for MEMS filters [193]. Four different types of silicon-based filter arrangements were shown comprising of both mechanical and electrostatic coupling. Enhanced rejection and bandwidth performance was demonstrated via combining coupling mechanisms with phase inversion techniques.

Typically, the coupling strength is kept very low to achieve a bandpass filter with low ripple effect. However, a strong mechanically coupled system comprising of two clamped-clamped beams with a coupling element attached to the mid-point (maximum velocity location) of the microbeams has been used for simultaneous filtering, mixing, and amplification $[182,194,195]$. The effect of the coupler location on the overall amplitude of the coupler as well as the microbeams was investigated using finite element modeling and experiments. A maximum transformation/amplification of resonator's amplitude from the midpoint of the microbeam to the midpoint of the coupler was achieved for similar dimensions of the microbeams and the coupler [182]. The excitation of combination 
resonances due to frequency mixing [196] was then utilized to achieve a tunable wide band filter [194]. A similar coupled structure modified to operate in air and perform the filtering operation was developed and tested [195].

Another filtering concept was presented in [5] where the hardening and softening behavior of two resonators is combined to achieve a filter with nearly flat passband and sharp roll-off characteristics. Two clamped-clamped microbeams with their outputs electrically coupled together are used. One of the microbeams is buckled using electrothermal stiffness modulation to achieve the softening behavior. The experiments showed a filter with flat passband and sharp roll-off.

Arrays of coupled resonators have been investigated both theoretically and experimentally for various applications [197, 198]. An integrated CMOS-MEMS free-free beam resonator array was demonstrated to have low motional impedance and high power handling capability [199]. It is demonstrated that having the mechanical coupling elements attached to the high velocity points helps in accentuating the desired modes and reject undesired spurious modes. An array of 9 resonators was demonstrated to improve the motional impedance and power handling capability by 10 times compared to a single resonator. Similarly, an array of 10 coupled free-free beams was experimentally demonstrated to improve the phase noise significantly [200]. In another work, an array of 7 corners coupled Polysilicon square plate resonators was also experimentally demonstrated to effectively reduce the series motional resistance of the resonator [201].

Arrays of coupled resonators are prone to fabrication variations and difficult to characterize due to the absence of electrical interconnects, particularly as the number of coupled elements increases. A unique scheme was proposed where a single input/output channel is used to characterize the entire resonator array for sensing applications [202]. In this scheme, an electrical resonator is coupled to an array of coupled resonators. To characterize the array in [203], they used inverse eigenvalue analysis, a perturbation analysis, and inverse problem theory, where eigenfrequencies from only one resonator (for a system of two coupled resonators) are required to reconstruct the system matrix to perform eigenvector extraction and full parameter extraction to sense mass/stiffness and coupling strength.

Distributed oscillatory systems such as coupled MEMS resonators exhibit interesting collective behavior, such as frequency locking, phase locking, and synchronization. It is due to these interesting nonlinear properties that recently there has been a focus on utilizing coupled M/NEMS resonators for neurocomputing [204-207]. These resonators are easy to fabricate and can potentially achieve enormous computing speeds when working in parallel. 
A canonical phase model approach was used to study the universal computational properties of oscillatory networks [204]. The study in [204] discusses three possible hardware implementations including "Phase-Locked Loop Neurocomputers," "Optical Neurocomputers," and "Mechanical Neurocomputers." This study identifies some of the major challenges faced in theoretical investigation of such systems.

In another study, a network of all-to-all coupled MEMS oscillators was investigated theoretically [205]. It was demonstrated that in the presence of stochasticity, the oscillator network is able to synchronize and store information in the relative phase differences. The study stated that using silicon-based MEMS oscillators for neurocomputing can potentially be robust against noise sources and fabrication imperfections.

Recently, an experimental demonstration of reservoir computing was presented utilizing the Duffing nonlinearity of a silicon beam combined with a delay-coupled reservoir architecture [207]. The system was shown to predict a time series and classify spoken words. Similarly, dynamics of MEMS coupled structures were also exploited to demonstrate continuous time recurrent neural networks (CTRNNs) [206]. The study in [206] utilizes the nonlinear dynamic phenomenon of bi-stability and hysteresis to simulate the detection and memory of a single rate model neuron. A straight beam was utilized to study the bi-stability, while the memory and detection is performed on an initially curved arch beam using the snap-through instability. The concept of electrical circuit resonance was used to trigger the bistability and amplify the resonator's response.

\subsection{Mode Localization in Coupled Resonators}

Over the past few years, the mode localization phenomenon in M/NEMS coupled resonators for sensing applications have gathered significant attention [208]. Mode localization refers to the confinement of vibrational energy to one of the modes of the coupled resonators as a result of a small perturbation in mass/stiffness of an unperturbed/balanced system. This can be visualized by looking at the frequency response of the two coupled resonators in Fig. 11. When a small perturbation (change in mass/stiffness in one of the resonator) is introduced to the unperturbed/balanced system, the vibrational energy is confined to the first mode of vibration. The strength of localization depends on the strength of the coupling and the magnitude of perturbation introduced to the coupled system. Another phenomenon that is closely related in coupled systems exhibiting mode localization is eigenvalue curve veering [209]. Figures 11a, b show the dynamic response of the coupled systems of Fig.10a represented by Eq. (2) and Eq. (3). It can be observed from Fig. 11b that the two eigenvalues approach each other but never intersect, and veer away as the stiffness of the 
first resonator is varied. This phenomenon is discussed in detail in the previous section for the case of inter-modal coupling of a single resonator. Here, a physical coupling either mechanical or electrostatic exists between the two resonators that is responsible for the veering behavior.

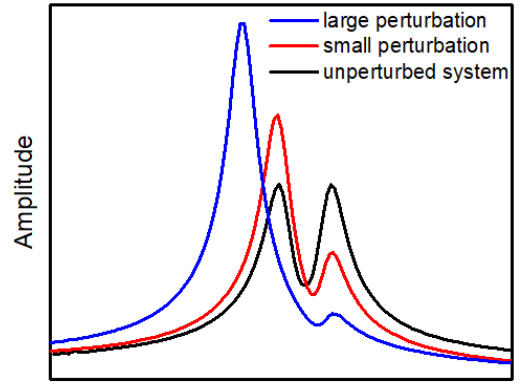

Frequency

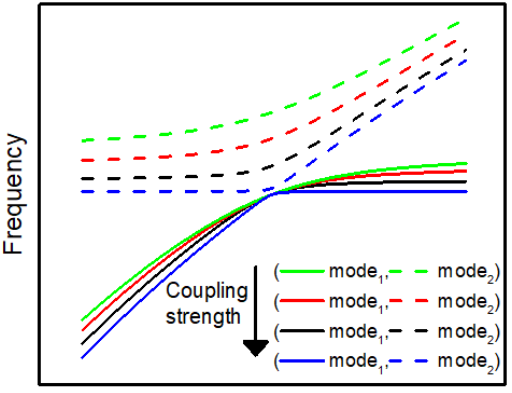

Stifness Perturbation

(a)

(b)

Fig.11. (a) The mode localization phenomenon for two coupled resonators. An increasing perturbation in the stiffness of one of the coupled resonators results in localization in the first mode. (b) The veering phenomenon in two coupled resonators. It can be observed that the eigenvalue curves of mode 1 and mode 2 approach each other as the coupling strength is decreased.

Commonly, a single M/NEMS resonator is used as a sensor based on the phenomenon of resonance frequency shift in response to an external stimulus (e.g., added mass or change in stiffness). Recently, it has been shown that utilizing two or more coupled structures exhibiting the phenomenon of mode localization, and measuring the change in the eigenstates/amplitude of the resonators, improves the sensitivity of the sensor by orders of magnitude higher than that of a single resonator based sensor [21]. A detailed review on the phenomenon of mode localization in MEMS resonators and its exploitation for sensing was published recently [21]. In this section, we will mainly focus on the advancements afterward.

At first, monitoring the change in eigenstates of mode localized sensor was used as an output signal [210]. Later, it was proposed that using the ratio between the quotients of the amplitudes of the two modes of a single resonator can be used for better sensitivity [211]. This method requires monitoring one resonator but at both modes of vibration. Both of these approaches have limitations in real-time sensing, which is imperative for practical application. Afterward, another sensing metric was proposed, which involves monitoring the ratio of the vibration amplitude of a single mode at both resonators [212]. Since only a single frequency is required to be monitored in this case, it 
potentially enables real-time sensing. It was recently demonstrated that the output metric of amplitude ratio depends on the input drive power variations [213]. To achieve high signal-to-noise ratios without compromising the operation of the sensor, it is recommended to operate near the veering zone where the stiffness and mass properties of the two resonators are closely matched. Reduction in conversion of the drive power noise to output noise is observed in this region, which can potentially enable high resolution measurements.

The proposed readout schemes of monitoring eigenstates or amplitude ratio have limited linear sensing regime due to the veering phenomenon of mode-localized sensors. Recently, algebraic relations of the eigenstates were proposed to improve the linear sensing performance of mode-localized sensors [214]. It was demonstrated both theoretically and experimentally that the algebraic sum of the amplitude ratios of two modes is a linear function of the stiffness perturbation with the largest nonlinearity error of $1.36 \%$. It was also demonstrated that the product of the amplitude ratios is approximately a constant. Same authors demonstrated enhancement of the linear sensing range using amplitude difference as a readout scheme for two coupled tuning fork resonators based stiffness sensor [215].
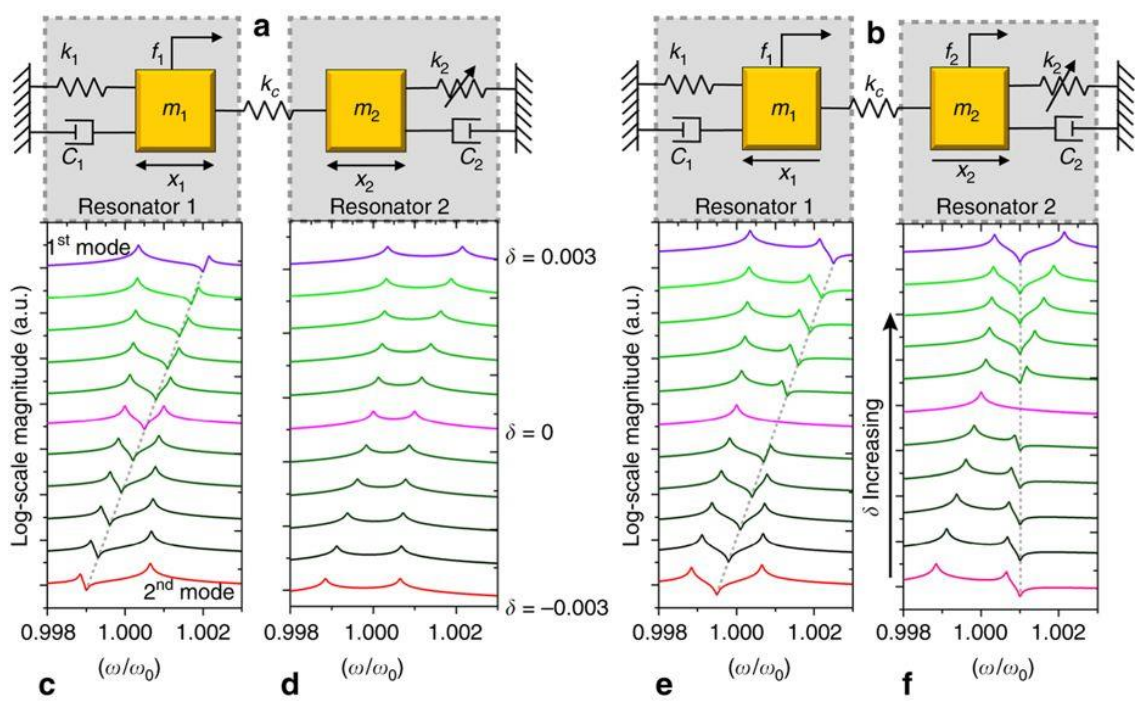

Fig. 12. (a) Model of disordered weakly coupled resonators under the single-resonator-driven (SRD) scheme. (b) Model of disordered weakly coupled resonators under the double-resonator-driven (DRD) scheme. (c) and (d) Responses of Resonator 1 and Resonator 2 with different stiffness perturbations under the SRD scheme. (e and f) Responses of Resonator 1 and Resonator 2 with different stiffness perturbations under the DRD scheme[216]. Reprinted with permission from [216]. 
In another recent study, two new characteristics of mode localization were demonstrated theoretically and experimentally [216], Fig. 12. First, it was shown that the anti-resonance loci vary linearly with the stiffness perturbation. Although tracking anti-resonance is not suitable as output sensing metric due to large measurement errors compared to tracking change in frequency or amplitude, it is significant as it can track the localization in the frequency domain and identify which resonator is perturbed. Second, it was demonstrated that driving both resonators instead of a single one can extend the linear sensing range at the cost of decrease in sensitivity (an order lower compared to a single-resonator-driven scheme).

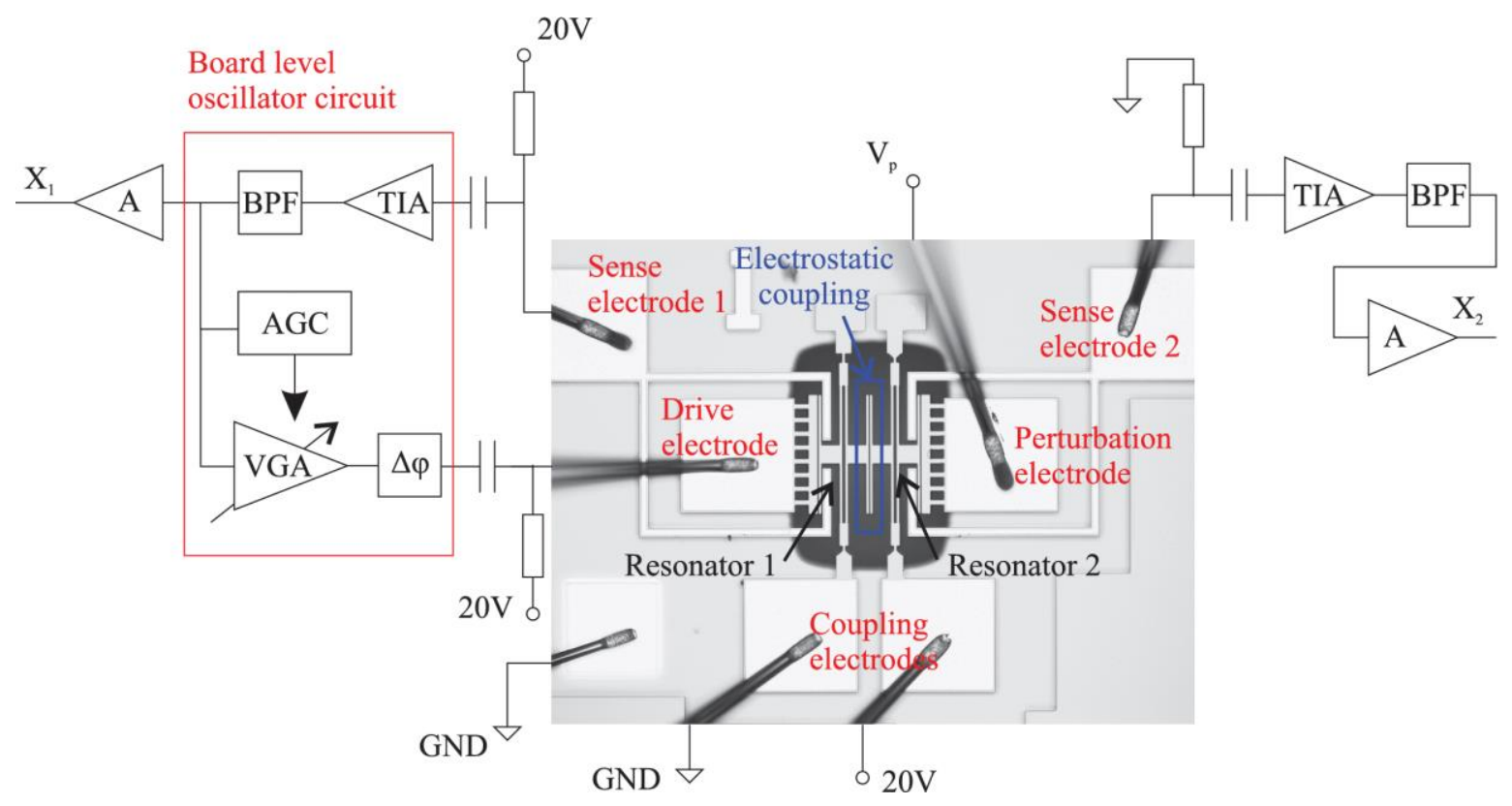

Fig. 13. Schematic showing closed-loop readout configuration for mode-localized resonant MEMS sensors. An optical micro-graph of the prototype mode-localized resonant sensor, i.e., a double-ended tuning fork resonator, is shown[217]. Reprinted with permission from [217].

Self-oscillation configurations of mode-localized sensors are also being explored for close-loop readout [217, 218], which is an important consideration for practical sensing devices. An experimental closed-loop readout configuration for mode-localized sensor based on electrostatically coupled resonators was demonstrated in [217], Fig. 13. The closed-loop results match fairly well with open-loop measurements. Similarly, a closed-loop accelerometer was experimentally demonstrated on three weakly coupled resonators [218]. 
Mode-localized sensors involve multi-degree-of-freedom systems, hence, each resonator in the array is subjected to same ambient environment (common mode), but localization happens only due to the induced perturbation on any of the resonator. Hence, such sensors can inherently take advantage of common mode reduction [219], and show resilience against ambient pressure and temperature drift. Commonly, single-degree-of-freedom sensors suffer from temperature and pressure fluctuations, which can be potentially eliminated using a mode-localized resonator. Ambient pressure drift rejection of a mode-localized sensor was experimentally demonstrated. A maximum error of amplitude ratio readout of $2.74 \%$ is experimentally demonstrated for a pressure range of $2.6-20 \mathrm{~Pa}$ [220]. In another experimental demonstration by same authors, temperature drift suppression for amplitude ratio readout was found to be $8.9 \%$ for a temperature range of $290-350 \mathrm{~K}[221]$.

Using amplitude related output metrics exhibit high sensitivity compared to the frequency shift detection, but suffer from low resolution due to the thermal noise and measuring circuit noise. Toward this, noise optimization strategies for mode-localized sensors were theoretically and experimentally investigated [222]. It was observed that coupling strength, quality factor, and stiffness mismatch between the two resonators are key parameters affecting the resolution of amplitude ratio measurements. It was also shown there is a trade-off between the resolution and the linearity of such sensors.

In another recent attempt to enhance the resolution of mode-localized resonators, a nonlinearity cancellation scheme has been experimentally demonstrated for two electrostatically coupled resonators, where the mechanical nonlinearity coming from the geometry of the resonators, and the electrostatic nonlinearity from the coupling cancel each other [223]. A four times increase in the output motional current in this regime was observed. It is also shown that using such a sensor operating in cancellation regime, the amplitude response for nonlinear actuation is 10 times higher than that of linear actuation.

Another important consideration for mode localized sensors is the quality factor of the coupled resonators, as it ultimately limits the sensitivity of the sensor. This adds additional requirement for such sensors to be encapsulated in vacuum. Recently, a mass sensor comprising of 3 degree-of-freedom coupled system and operating in air was theoretically and experimentally demonstrated [224]. However, a minimum detectable mass of only 1.9ng was reported in air. A comparison of the three output metrics was also carried out: resonance frequency shift, vibration amplitude change, and amplitude ratio. It was concluded that amplitude ratio still yields highest sensitivity. 
Ideally, identical resonators are coupled to realize the mode-localized sensors. However, process induced variability is unavoidable in M/NEMS systems and can impact significantly the performance of sensors based on coupled resonators. A theoretical and experimental investigation into the sensitivity degradation of coupled resonators due to induced variability was presented in [225]. It was concluded that eigenvector based output metrics are more severely affected by process-induced variabilities. One way to compensate for these variations is tuning the stiffness of the resonator by external DC or electrothermal loading.

Sensor based mode localization was first demonstrated for mass sensing using two mechanically coupled cantilever beams [210]. The authors demonstrate both theoretically and experimentally that the relative change in the eigenstates magnitude is larger by a factor of 2 over the relative change in the eigenfrequencies. Same authors also showed that the sensitivity in such sensors can be increased when using an array of resonators [226]. An array of 15 mechanically coupled cantilevers was shown to improve the sensitivity by two to three orders over two coupled cantilevers of [210]. A mass sensor has been recently demonstrated to operate in ambient pressure using the mode-localization phenomenon [224].

A force sensor based on three electrostatically coupled resonators was also demonstrated theoretically and experimentally [227]. The authors also theoretically estimated the noise floor for the amplitude ratio output metric using a transfer function model.

Mode-localized sensors have also been proposed as charge sensors or electrometers [228, 229]. A pair of weakly coupled double-ended tuning fork resonators was used to experimentally demonstrate charge sensing. Eigenstate changes were measured to estimate the charge input. In another study, a similar structure was used to demonstrate an electrometer [229].

Additionally, several MEMS accelerometers were proposed based on the phenomenon of mode localization [218, 230232]. Amplitude ratio shift was used to measure the input acceleration onto two weakly coupled double-ended tuning fork resonators [231]. Open loop measurements were conducted to show resolution of $0.619 \mathrm{mg}$ and a nonlinearity of 3.5\%. Same authors presented another accelerometer design based on single-tine tuning fork resonators in a closed loop circuit arrangement [230]. Similarly, three weakly coupled resonators were also used as accelerometers and a resolution of $1.1 \mu \mathrm{g} / \sqrt{ } \mathrm{Hz}$ is reported [218]. In another study, a novel structure design was used as an accelerometer and a $7 \mu \mathrm{g}$ bias stability was achieved, which is almost two time higher than the state of the art [232]. A mechanical 
coupling element was used in this work however it was proposed that electrostatic coupling is better choice for high resolution and long integrations times. It also reduces temperature dependence of the coupler and allows for optimization in coupling stiffness and sensitivity of the device.

As discussed, considerable effort has been made towards developing mode localized sensors. However, such applications of mode localization are still relatively new, and there still lacks a thorough understanding of the localization phenomenon in such systems; especially complex ones involving non-uniform geometries and complicated transduction methods. Mode localized sensors require high quality factors for higher sensitivity; hence, operating in high damping media could affect the sensitivity. Therefore, effect of utilizing different damping conditions under the different elements of coupled system are worth investigation. Investigation localization at the higher order modes of coupled systems is another area of future potential. This can enable simultaneous sensing/monitoring of different parameters, which could potentially contribute towards developing sophisticated and highly sensitive sensors. Further, most of the applications based on localization operate in the linear regime. However, operating these devices in the nonlinear regime can reveal richer dynamics, which can be used for enhancing the existing sensors' performance or developing new applications.

\section{Nonlinear Response of MEMS Resonators using Perturbation Methods}

Perturbation methods are powerful mathematical tools to analyze the response of various MEMS devices; particularly MEMS resonators. Basically, one aims at applying perturbation on the governing equations of the system to obtain approximate analytical solutions that provide analytical depth and convenience, reduce the computational cost, and provide additional information, such as the stability of the system. Because of the fact that MEMS have many sources of nonlinearity, especially the electrostatic actuation, in addition to the environmental and geometrical sources [84], which increase the complexity of the system; using perturbation methods in the analysis can be very useful.

There has been considerable research on the application of perturbation methods on MEMS. For instance, the Homotopy perturbation method has been used to analyze the static motion of MEMS resonators and their pull-in characteristics [233-235]. Singular perturbations have been used for complex MEMS models, such as for membranes and thin plates, to obtain closed-form solutions for the electrostatic deflection [236-239] or to account for imperfections [240] in MEMS comb drive. Singular perturbation methods were also used to analyze the influence of 
squeeze film [241-243] and thermoelastic damping [244-247]. The study of Casimir forces on MEMS is another aspect where perturbation methods have been used [248, 249].

Perturbation methods have been particularly used to investigate the dynamics of MEMS resonator and to understand the influence of the different nonlinearities in the system. These include the multiple time scales (MTS), averaging, and the Lindstedt Poincare methods. MEMS resonators are investigated when subjected to parametric, primary, or secondary excitations, or any combination of these. Several works have been presented to analyze the dynamics under parametric excitation using perturbation methods in gyroscopes [250, 251], comb drives fingers [252-255], bridge resonators [256], and microbeams [257-260]. Primary-resonance excitation has been also of great interest. Perturbation methods are applied to obtain the modulation equations that govern the amplitude and phase response of the resonator. Examples of these studies can be found in microbeams [107, 166, 217, 261-279], torsional mirrors [280, 281], self-excited resonators [282-285], PZT based MEMS models [286-288], MEMS filters [171], MEMS arch resonators [169, 289], carbon Nanotubes [290], and coupled oscillators [291]. The influence of secondary-resonance excitations on MEMS has been also studied. Examples of these can be found in AFM [292], MEMS microbeam resonators [293-297], mechanically coupled resonators [298], and ring MEMS resonators [299]. In most of these applications, a lumped parameter model is employed to study the response while considering Taylor series expansion of the electrostatic nonlinearity. More theoretical aspects related to the use of perturbations on MEMS resonator using lumped parameters can be found in [300-302].

In this part of the review, we highlight important aspects on the use of MTS method to characterize the nonlinear response of MEMS resonators. In particular, the influence of the combined effects of secondary and primary excitations are considered due to the application of moderate to high AC voltages with two source excitations in comparison to previously published works focusing on a single term excitation. In Sec. 5.1, we present the scaling method of the two-excitation terms using lumped parameter modelling considering different resonance cases. In addition, in Sec. 5.2, we demonstrate how to take into account the influence of the secondary excitation term on the two-to-one internal resonance using the MTS perturbation method.

\subsection{MEMS resonators Under Primary and Secondary Excitations}




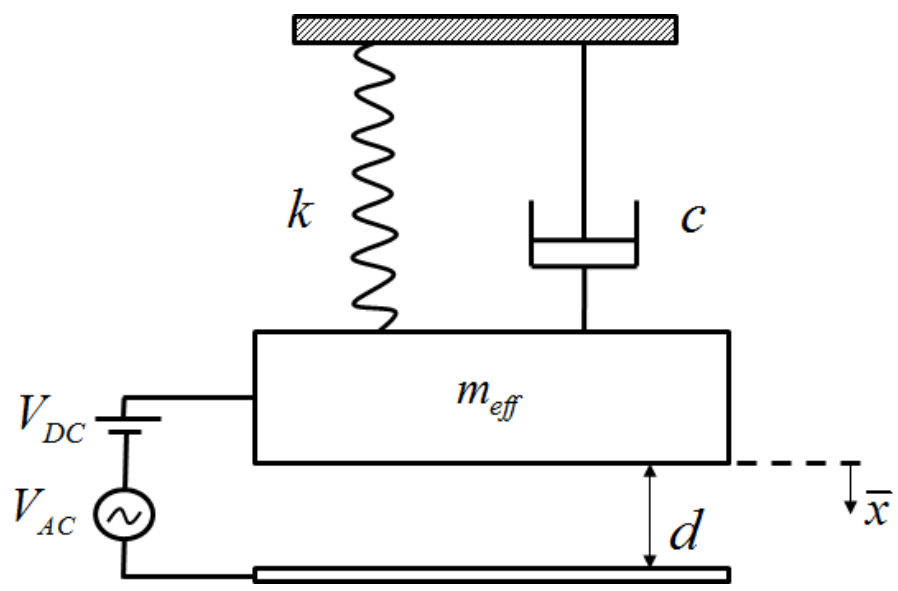

Fig.14. Schematic of a lumped parameter model of an electrostatically actuated MEMS resonator.

Next, we demonstrate the use of the method of multiple time scales on an electrostatically actuated MEMS resonator. The lumped parameter system that describes the displacement $\bar{x}(t)$ of a generic MEMS resonator, Fig. 14, with Duffing type nonlinearity under electrostatic loading can be written as [84]

$m_{e f f} \ddot{\bar{x}}+c \dot{\bar{x}}+k \bar{x}+\bar{\alpha}_{c} \bar{x}^{3}=\frac{A \varepsilon_{0}\left(V_{D C}+V_{A C} \cos (\Omega \hat{t})\right)^{2}}{2(d-\bar{x})^{2}}$

where the dot denotes the derivative with respect to time $\hat{t}, m_{\text {eff }}$ is the effective mass, $c$ is damping coefficient, $k$ is the stiffness, $\bar{\alpha}_{c}$ is the cubic nonlinearity coefficient, $A$ is the area of the electrode, and $d$ is the gap between the resonator and the lower electrode. Next, we nondimensionalize Eq. (6) by introducing $x=\frac{\bar{x}}{d}$ and $t=\sqrt{\frac{k}{m_{\text {eff }}}} \hat{t}$, which yields

$\ddot{x}+\mu \dot{x}+x+\alpha_{c} x^{3}=\frac{\beta\left(V_{D C}+V_{A C} \cos (\Omega t)\right)^{2}}{(1-x)^{2}}$

where $\mu=\sqrt{\frac{c^{2}}{k m_{e f f}}} ; \alpha_{c}=\frac{d^{2} \bar{\alpha}_{c}}{k} ; \beta=\frac{A \varepsilon_{0}}{2 d^{3} k}$. 
The type of electrostatic loading, i.e., large $V_{D C}$ or large $V_{A C}$ or both, can result into excitations, which can be erroneously neglected. To clarify this, we expand the electrostatic force term as

$$
\frac{\beta\left(V_{D C}+V_{A C} \cos (\Omega t)\right)^{2}}{(1-x)^{2}}=\beta \frac{2 V_{A C} V_{D C} \cos (\Omega t)}{(1-x)^{2}}+\beta \frac{V_{A C}^{2} \cos (2 \Omega t)}{2(1-x)^{2}}+\beta \frac{V_{D C e f f}}{(1-x)^{2}}
$$

where $V_{D C \text { eff }}=V_{D C}^{2}+\frac{1}{2} V_{A C}^{2}$. It is noticed that the $V_{A C}$ term produces a static and a dynamic term that influences the linear and nonlinear forced vibration problem. Substituting Eq. (8) into Eq. (7) yields

$$
\ddot{x}+\mu \dot{x}+x+\alpha_{c} x^{3}=\beta \frac{2 V_{A C} V_{D C} \cos (\Omega t)}{(1-x)^{2}}+\beta \frac{V_{A C}^{2} \cos (2 \Omega t)}{2(1-x)^{2}}+\beta \frac{V_{D C e f f}}{(1-x)^{2}}
$$

The static part of Eq. (9), $\delta$, is obtained by dropping the time dependent terms, which yields

$$
\delta+\alpha_{c} \delta^{3}=\beta \frac{V_{D C e f f}}{(1-\delta)^{2}}
$$

Equation (10) is solved algebraically to obtain the static response. Next, we perturb the dynamics around the static solution, i.e. $x(t)=\delta+u(t)$, and expand the electrostatic term in Taylor series around the static solution to obtain

$$
\begin{aligned}
\ddot{u}+\mu \dot{u}+\omega^{2} u+\alpha_{q} u^{2} & +\alpha_{c e f f} u^{3}=\beta \frac{2 V_{A C} V_{D C} \cos (\Omega t)}{(1-\delta)^{2}}+\beta \frac{V_{A C}^{2} \cos (2 \Omega t)}{2(1-\delta)^{2}}+2 u\left(\beta \frac{2 V_{A C} V_{D C} \cos (\Omega t)}{(1-\delta)^{3}}+\beta \frac{V_{A C}^{2} \cos (2 \Omega t)}{2(1-\delta)^{3}}\right) \\
& +3 u^{2}\left(\beta \frac{2 V_{A C} V_{D C} \cos (\Omega t)}{(1-\delta)^{4}}+\beta \frac{V_{A C}^{2} \cos (2 \Omega t)}{2(1-\delta)^{4}}\right)+4 u^{3}\left(\beta \frac{2 V_{A C} V_{D C} \cos (\Omega t)}{(1-\delta)^{5}}+\beta \frac{V_{A C}^{2} \cos (2 \Omega t)}{2(1-\delta)^{5}}\right)
\end{aligned}
$$

where

$\omega^{2}=1+3 \delta^{2} \alpha_{c}-2 \frac{\beta V_{D C \text { eff }}}{(1-\delta)^{3}} ; \alpha_{q}=3 \delta \alpha_{c}-\beta \frac{3 V_{D C \text { eff }}}{(1-\delta)^{4}} ; \alpha_{c e f f}=\alpha_{c}-\beta \frac{4 V_{D C e f f}}{(1-\delta)^{5}}$

From Eq. (11), we observe that the natural frequency of the resonator is shifted due to the static response under competing effects of the Duffing coefficient, producing $3 \delta^{2} \alpha_{c}$ term, and the electrostatic term. In addition, a new quadratic nonlinearity is introduced due to the influence of the electrostatic response. This signifies the importance of the expansion in revealing the effective nonlinearities due to the electrostatic term. In addition, it was revealed in [303] 
that the Taylor expansion of the electrostatic term yields the same dynamic response to that of the cross-multiplied one up to the same order using perturbations. To further analyze the dynamic response, we analyze Eq. (11) using MTS to study the effect of different forcing terms and different types of nonlinearities. The response of the resonator, $u(t)$, is assumed to follow an auto-scaling defined by

$u(t)=\grave{o} u_{1}\left(T_{0}, T_{1}, T_{2}\right)+\grave{o}^{2} u_{2}\left(T_{0}, T_{1}, T_{2}\right)+\grave{o}^{3} u_{3}\left(T_{0}, T_{1}, T_{2}\right)+\mathrm{O}\left(\grave{o}^{4}\right)$

where $\dot{o}$ is introduced as a bookkeeping parameter to segregate the scales of different nonlinearities. $T_{i}$ are the different time scales defined by $T_{i}=\grave{o}^{i} t$. The expansion is up to three orders to account for the influence of the cubic nonlinearity. In what follows, we consider the case when the frequency of excitation is near the natural frequency, i.e. $\Omega \approx \omega$, and the case when the frequency of excitation is near half the natural frequency, i.e. superharmonic excitation $\Omega \approx 1 / 2 \omega$

\subsubsection{Excitation near the natural frequency}

In this case, the term $\cos (2 \Omega t)$ is scaled at $\grave{o}$ to obtain the particular solution at the first order. The term $\cos (\Omega t)$ is scaled at the third order level $\grave{o}^{3}$ while damping is scaled at $\grave{o}$ to damp the response obtained at the quadratic level. Substituting Eq. (12) into Eq. (11), while considering the scales, yields

$$
\begin{aligned}
& \dot{o}^{1}: D_{0}^{2} u_{1}+\omega^{2} u_{1}=F 1_{2 \Omega} \cos (2 \Omega t) \\
& \grave{o}^{2}: D_{0}^{2} u_{2}+\omega^{2} u_{2}=-2 D_{0} D_{1} u_{1}-\alpha_{q} u_{1}^{2}-\mu D_{0} u_{1}+F 2_{2 \Omega} u_{1} \cos (2 \Omega t) \\
& \dot{o}^{3}: D_{0}^{2} u_{3}+\omega^{2} u_{3}=-2 D_{0} D_{1} u_{2}-\mu D_{1} u_{1}-\mu D_{0} u_{2}-2 D_{0} D_{2} u_{1}-D_{1}^{2} u_{1}-2 \alpha_{q} u_{2} u_{1}-\alpha_{\text {ceff }} u_{1}^{3}+ \\
& \quad F 2_{2 \Omega} u_{2} \cos (2 \Omega t)+F 3_{2 \Omega} u_{1}^{2} \cos (2 \Omega t)+F 1_{\Omega} \cos (\Omega t)
\end{aligned}
$$

where $D_{i}^{j}=\frac{\partial^{j}}{\partial T_{i}^{j}} ; F 1_{2 \Omega}=\beta \frac{V_{A C}^{2}}{2(1-\delta)^{2}} ; F 2_{2 \Omega}=\beta \frac{V_{A C}^{2}}{(1-\delta)^{3}} ; F 3_{2 \Omega}=\beta \frac{3 V_{A C}^{2}}{2(1-\delta)^{4}} ; F 1_{\Omega}=\beta \frac{2 V_{A C} V_{D C}}{(1-\delta)^{2}}$.

Following the details of the multiple time scales perturbations method and using the method of reconstitution, we end up with two modulation equations that govern the amplitude $a$ and phase $\gamma$ of the response [304].

The dynamic solution is then written as 


$$
\begin{gathered}
u(t)=a \cos (\Omega t+\gamma)+\frac{a^{2}}{2} \Lambda_{2 \omega} \cos (2 \Omega t+2 \gamma)+2 \Lambda_{2 \Omega} \cos (2 \Omega t)+2 \Lambda_{2 \Omega 2} \sin (2 \Omega t)- \\
a \Lambda_{\omega+2 \Omega} \cos (3 \Omega t+\gamma)-2 \Lambda_{4 \Omega} \cos (4 \Omega t)+\frac{F 2_{2 \Omega} \Lambda_{2 \Omega}}{\omega^{2}}-\frac{a^{2} \alpha_{q}}{2 \omega^{2}}-\frac{2 \Lambda_{2 \Omega}^{2} \alpha_{q}}{\omega^{2}}
\end{gathered}
$$

where $\sigma_{1}$ is a frequency detuning parameter, $\Lambda_{4 \Omega}=\frac{\Lambda_{2 \Omega}\left(F 2_{2 \Omega}-2 \alpha_{q} \Lambda_{2 \Omega}\right)}{2\left(-\omega^{2}+16 \Omega^{2}\right)} \cdot \Lambda_{2 \Omega}, \Lambda_{2 \Omega 2}, \Lambda_{2 \omega}, \Lambda_{\omega+2 \Omega}$ are the coefficients pertaining to the particular solution defined by

$$
\Lambda_{2 \Omega}=\frac{F 1_{2 \Omega}}{2\left(\omega^{2}-4 \Omega^{2}\right)} ; \Lambda_{2 \Omega 2}=\frac{2 \mu \Omega \Lambda_{2 \Omega}}{\omega^{2}-4 \Omega^{2}} ; \Lambda_{2 \omega}=\frac{\alpha_{q}}{3 \omega^{2}} ; \Lambda_{\omega+2 \Omega}=\frac{\left(F 2_{2 \Omega}-4 \alpha_{q} \Lambda_{2 \Omega}\right)}{8 \Omega(\omega+\Omega)}
$$

It is noted from Eq. (14) that the response due to the $2 \Omega$ term does not depend only on the quadratic nonlinearity but also on the direct excitation term.

\subsubsection{Excitation at half the natural frequency}

In this case, we consider the excitation frequency near half the natural frequency, i.e. $\Omega \approx 1 / 2 \omega$. The term $\cos (2 \Omega t)$ is scaled at $\hat{o}^{3}$ because it becomes a primary resonance excitation. The term $\cos (\Omega t)$ is scaled at the first order level $\grave{o}$ to obtain the particular solution while damping is scaled at $\grave{o}$ to damp the response obtained at the quadratic level. Substituting Eq. (12) while considering the new scales yields

$$
\begin{aligned}
& \dot{o}^{1}: D_{0}^{2} u_{1}+u_{1} \omega^{2}=F 1_{\Omega} \cos (\Omega t) \\
& \dot{o}^{2}: D_{0}^{2} u_{2}+u_{2} \omega^{2}=-\mu D_{0} u_{1}-2 D_{0} D_{1} u_{1}-\alpha_{q} u_{1}^{2}+F 2_{\Omega} u_{1} \cos (\Omega t) \\
& \dot{o}^{3}: D_{0}^{2} u_{3}+\omega^{2} u_{3}=-\mu D_{0} u_{2}-\mu D_{1} u_{1}-D_{1}^{2} u_{1}-\alpha_{c e f f} u_{1}^{3}-2 D_{0} D_{2} u_{1}-2 \alpha_{q} u_{2} u_{1}+F 1_{2 \Omega} \cos (2 \Omega t)+ \\
& \quad F 2_{\Omega} u_{2} \cos (\Omega t)+F 3_{\Omega} u_{1}^{2} \cos (\Omega t)
\end{aligned}
$$

where $F 2_{\Omega}=\beta \frac{4 V_{A C} V_{D C}}{(1-\delta)^{3}} ; F 3_{\Omega}=\beta \frac{6 V_{A C} V_{D C}}{(1-\delta)^{4}}$. Following the multiple time scales method and using the method of reconstituting, we ended up with two modulation equations [304]. The final dynamic solution is

$$
\begin{aligned}
u(t)= & a \cos (2 \Omega t+\gamma)+\Lambda_{2 \omega} \frac{a^{2}}{2} \cos (2 \Omega t+2 \gamma)+2 \Lambda 1_{\Omega} \cos (\Omega t)-2 \Lambda 2_{\Omega} \sin (\Omega t) \\
& +\Lambda_{\omega-\Omega} a \cos (\Omega t+\gamma)-\Lambda_{\omega+\Omega} a \cos (3 \Omega t+\gamma)+\frac{F 2_{\Omega} \Lambda 1_{\Omega}}{\omega^{2}}-\frac{2 \Lambda 1_{\Omega}^{2} \alpha_{q}}{\omega^{2}}-\frac{a^{2} \alpha_{q}}{2 \omega^{2}}
\end{aligned}
$$


where $\Lambda 1_{\Omega}, \Lambda 2_{\Omega}, \Lambda_{\omega+\Omega}, \Lambda_{\omega-\Omega}, \Lambda_{2 \omega}$ are coefficients of the particular solutions defined by

$$
\Lambda 1_{\Omega}=\frac{F 1_{\Omega}}{2\left(\omega^{2}-\Omega^{2}\right)} ; \Lambda 2_{\Omega}=\frac{\mu \Omega \Lambda 1_{\Omega}}{\Omega^{2}-\omega^{2}} ; \Lambda_{\omega+\Omega}=\frac{\left(F 2_{\Omega}-4 \alpha_{q} \Lambda 1_{\Omega}\right)}{2 \Omega(2 \omega+\Omega)} ; \Lambda_{\omega-\Omega}=\frac{\left(F 2_{\Omega}-4 \alpha_{q} \Lambda 1_{\Omega}\right)}{2(2 \omega-\Omega) \Omega} ; \Lambda_{2 \omega}=\frac{\alpha_{q}}{3 \omega^{2}}
$$

It is observed from the dynamics response solution that there exists a direct excitation component in addition to the excitation induced by the nonlinearity. In addition, we notice contribution from the higher order secondary harmonics.

To validate the solution presented in each case we plot and compare the MTS solution with the solution obtained by integrating in time the original system of Eq. (7). The results are shown in Fig. 15.

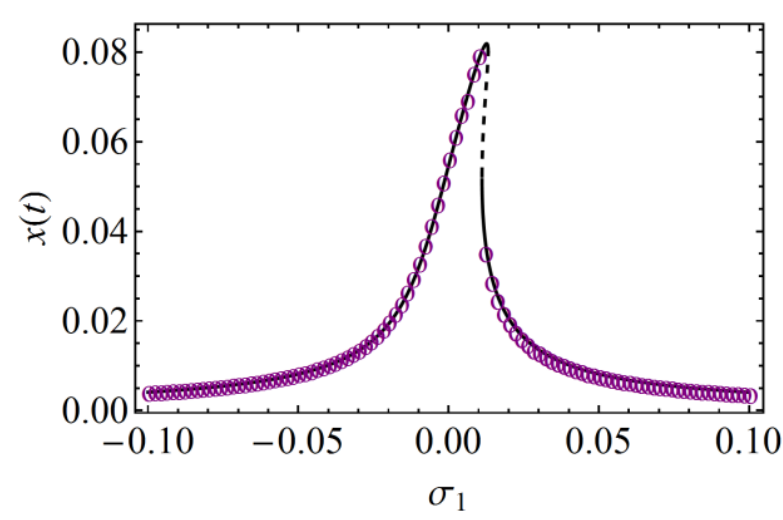

(a)

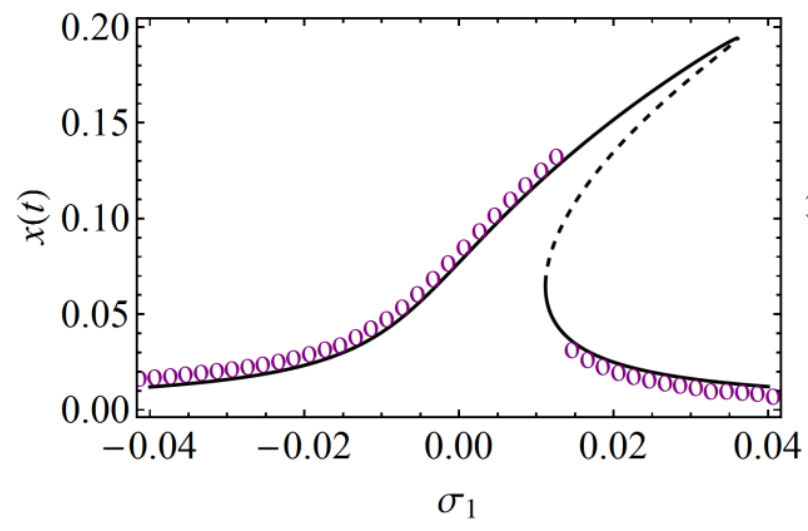

(c)

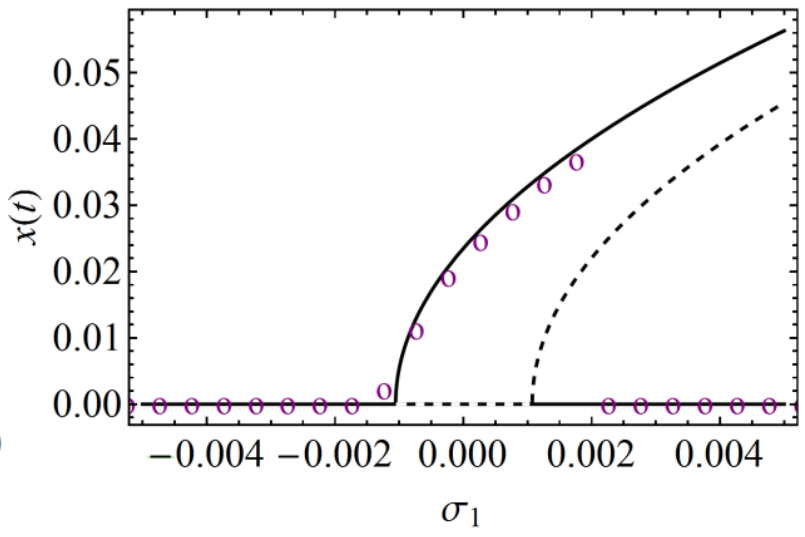

(b)

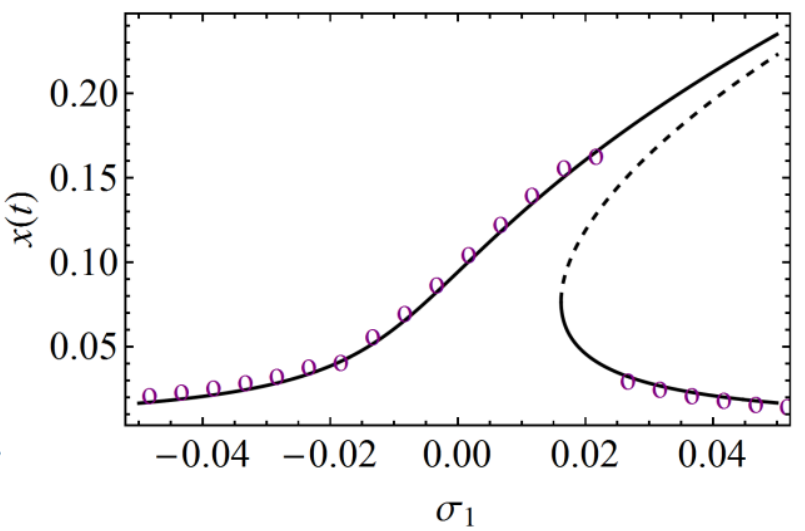

(d)

Fig.15. Frequency response curves of the microstructure at different excitation conditions, $\beta=4.08419 \times 10^{-4}, \alpha_{c}=5.07264$. (O) Solution from forward sweep in long time integration of Eq. (7). (—) Multiple time scales stable solution. (- - - -) Multiple 
time scales unstable solution. (a)Primary excitation with weak subharmonic: $\Omega \approx \omega, \mu=0.01, V_{D C}=1, V_{A C}=1$. (b) Pure subharmonic excitation: $\Omega \approx \omega, \mu=0.0025, V_{D C}=0, V_{A C}=4$. (c) Superharmonic excitation leading to primary excitation: $\Omega \approx \omega / 2, \mu=0.01, V_{D C}=3, V_{A C}=3$. (d) Primary excitation with strong subharmonic: $\Omega \approx \omega, \mu=0.01, V_{D C}=1, V_{A C}=4$.

The results in Fig. 15 are generated for the clamped-clamped resonator in [305]. An overall good agreement is observed between the MTS solution and the long-time integration one. In addition, the multiple scales is able to predict the subharmonic response features observed in Fig. 15b. In the latter cases in Fig. 15c and Fig. 15d the response of the system is mainly due to the direct excitation of the system via doubling the superharmonic excitation, which yields a primary resonance that dominates the response of the system.

\subsection{MEMS Resonators in Internal Resonances}

Perturbations methods have been applied on the governing equations of MEMS resonators to study different aspects related to internal resonances. Examples of these studies are three-to-one internal resonance in microbeams [306], two-to-one [32, 307], three-to-one [308, 309], and one-to-one internal resonance [309] in MEMS arch beams, two-toone internal resonance between the flexural and torsional mode of T-shaped resonator [310], two-to-one internal resonance in microscanners [160], and three-to-one internal resonance in PZT based MEMS resonator [311]. In all of these studies the MTS or averaging are utilized considering the modal coupling throughout the nonlinear system to study the energy exchange during the interaction.

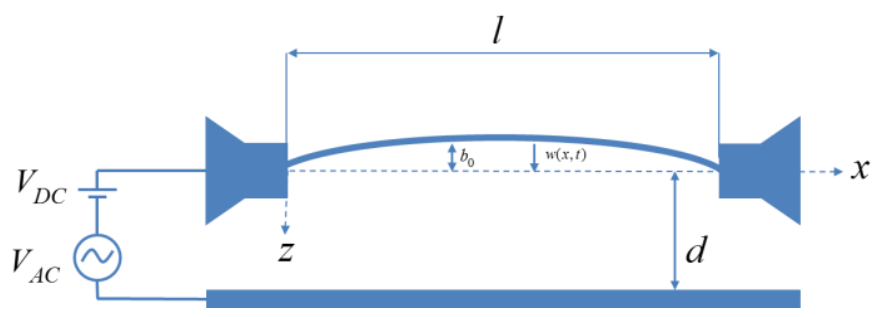

Fig.16. Schematic of a clamped-clamped arch beam under electrostatic actuation. 
In this section, we demonstrate the application of the multiple time scales to analyze internal resonances in MEMS. We consider the direct attack approach in which we apply the perturbation method directly on the partial differential equation. Moreover, the linear mode shapes and the particular solutions pertaining to the MTS procedure have spatial distribution that depends on the electrostatic voltage. For this case, the two-to-one internal resonance in MEMS arch resonator [307], Fig. 16, is illustrated as an example where the nondimensional equation of motion of the MEMS resonator is given by

$\frac{\partial^{2} w}{\partial t^{2}}+\frac{\partial^{4} w}{\partial x^{4}}+c \frac{\partial w}{\partial t}=\left(\left(\frac{\partial^{2} w}{\partial x^{2}}+\frac{d^{2} w_{0}}{d x^{2}}\right)\left[N+\alpha_{1} \int_{0}^{1}\left[\frac{\partial w^{2}}{\partial x}+2 \frac{\partial w}{\partial x} \frac{d w_{0}}{d x}\right] d x\right]\right)+\alpha_{2} \frac{\left(V_{D C}+V_{A C} \cos (\Omega t)\right)^{2}}{\left(1-w-w_{0}\right)^{2}}$

subjected to the boundary conditions

$w(0, t)=w(1, t)=0$ and $\left.\frac{\partial w}{\partial x}\right|_{(0, t)}=\left.\frac{\partial w}{\partial x}\right|_{(1, t)}=0$

where $w$ is the transverse deflection, $x$ is the position along the arch, $t$ is the time, $N$ is the axial force (can be tuned from electrothermal actuation), $w_{0}$ is the initial deflection of the arch, and the coefficients in Eq. (19) are defined by

$\alpha_{1}=6 \frac{d^{2}}{h^{2}} ; \quad N=\frac{l^{2}}{E I} \hat{N} ; \quad \alpha_{2}=\frac{6 \varepsilon l^{4}}{E h^{3} d^{3}} ; \quad c=\frac{l^{4}}{E I T} \hat{c} ; \quad \Omega=T \hat{\Omega}$

where $d$ is the gap, $h$ is beam thickness, $l$ is the beam length, $E$ modulus of elasticity, $I$ is area moment of inertia, $\varepsilon$ is the die electric constant, and $T$ is the nondimensional period. Depending on the magnitude of excitation, the influence of the $V_{A C}$ forcing term on the static and dynamic parts is analyzed by expanding the electrostatic term and considering

$V_{E f f D C}=V_{D C}{ }^{2}+\frac{1}{2} V_{A C}{ }^{2}$ and $V(t)=2 V_{A C} V_{D C} \cos (\Omega t)+\frac{1}{2} V_{A C}{ }^{2} \cos (2 \Omega t)$

Then, the static deflection $w_{s}(x)$ of the device due to the applied electrostatic load is written as

$$
\frac{d^{4} w_{s}}{d x^{4}}-N \frac{d^{2} w_{s}}{d x^{2}}=\left(\left(\frac{d^{2} w_{s}}{d x^{2}}+\frac{d^{2} w_{s a}}{d x^{2}}\right) \alpha_{1} \int_{0}^{1}\left[\left(\frac{d w_{s}}{d x}\right)^{2}+2 \frac{d w_{s}}{d x} \frac{d w_{s a}}{d x}\right] d x\right)+\alpha_{2} \frac{V_{E f f D C}}{\left(1-w_{s}-w_{s a}\right)^{2}}
$$


where $w_{s a}$ is the static deflection due to the initial rise and the applied axial load. The static equation can be solved by either the shooting method [306] or via Galerkin method utilizing beam mode shapes [34]. The linear eigenvalue problem under the influence of the electrostatic load is written as

$$
\frac{\partial^{2} w}{\partial t^{2}}+\frac{\partial^{4} w}{\partial x^{4}}-N \frac{\partial^{2} w}{\partial x^{2}}=\left(\left(\frac{d^{2} w_{s}}{d x^{2}}\right) \alpha_{1} \int_{0}^{1}\left[2 \frac{\partial w}{\partial x} \frac{d w_{s}}{d x}\right] d x+\left(\frac{\partial^{2} w}{\partial x^{2}}\right) \alpha_{1} \int_{0}^{1}\left(\frac{\partial w_{s}}{\partial x}\right)^{2} d x+\right)+2 \alpha_{2} \frac{V_{E f f D C}}{\left(1-w_{s}\right)^{3}} w
$$

We solve Eq. (24) using the Galerkin procedure in [34] to obtain the mode shapes and frequencies of the resonator. Then, we perturb Eq. (19) around the equilibrium configuration and expand the electrostatic term in Taylor series, which yield

$$
\begin{aligned}
\frac{\partial^{2} w}{\partial t^{2}}+\frac{\partial^{4} w}{\partial x^{4}}+c \frac{\partial w}{\partial t}-N \frac{\partial^{2} w}{\partial x^{2}} & =\left(\left(\frac{\partial^{2} w}{\partial x^{2}}+\frac{d^{2} w_{s}}{d x^{2}}\right) \alpha_{1} \int_{0}^{1}\left[\left(\frac{\partial w}{\partial x}\right)^{2}+2 \frac{\partial w}{\partial x} \frac{d w_{s}}{d x}\right] d x\right)+\alpha_{2} \frac{V(t)}{\left(1-w_{s}\right)^{2}} \\
& +2 \alpha_{2} \frac{\left[V_{E f f D C}+V(t)\right]}{\left(1-w_{s}\right)^{3}} w+3 \alpha_{2} \frac{\left[V_{E f D C}+V(t)\right]}{\left(1-w_{s}\right)^{4}} w^{2} \\
& +4 \alpha_{2} \frac{\left[V_{E f f D C}+V(t)\right]}{\left(1-w_{s}\right)^{5}} w^{3}+H O T
\end{aligned}
$$

where HOT denotes higher order terms. In this case, we observe that both terms $\cos (\Omega t)$ and $\cos (2 \Omega t)$ directly excite the modes participating in the two-to-one internal resonance. Therefore, we scale the forcing terms at the quadratic level along with damping and we consider the solution in multiple time scales expanded up to third orders as

$$
w(x, t ; \varepsilon)=\sum_{j=1}^{3} \varepsilon^{j} w_{j}\left(x, T_{0}, T_{1}, T_{2}\right)
$$

Substituting the expansion of Eq. (26) into Eq. (25) yields

$$
\begin{aligned}
& \varepsilon^{1}: \ell\left(w_{1}\right)=D_{0}^{2} w_{1}+w_{1}^{i v}-N w_{1}^{\prime \prime}-2 \alpha_{1} w_{s}^{\prime \prime}\left(\int_{0}^{1} w_{s}^{\prime} w_{1}^{\prime} \mathrm{d} x\right)-\frac{2 \alpha_{2} V_{E f D C}}{\left(1-w_{s}\right)^{3}} w_{1}=0 \\
& \varepsilon^{2}: \ell\left(w_{2}\right)=-2 D_{0} D_{1} w_{1}-c D_{0} w_{1}+\alpha_{1} w_{s}^{\prime \prime}\left(\int_{0}^{1}\left(w_{1}^{\prime}\right)^{2} \mathrm{~d} x\right)+2 \alpha_{1} w_{1}^{\prime \prime}\left(\int_{0}^{1} w_{s}^{\prime} w_{1}^{\prime} \mathrm{d} x\right)+\frac{3 \alpha_{2} V_{E f D C}}{\left(1-w_{s}\right)^{4}} w_{1}^{2}+\frac{2 \alpha_{2} V_{A C} V_{D C}}{\left(1-w_{s}\right)^{2}} \cos \left(\Omega T_{0}\right) \\
& +\frac{\alpha_{2} V_{A C}^{2}}{2\left(1-w_{s}\right)^{2}} \cos \left(2 \Omega T_{0}\right)
\end{aligned}
$$




$$
\begin{gathered}
\varepsilon^{3}: \ell\left(w_{3}\right)=-2 D_{0} D_{2} w_{1}-2 D_{0} D_{1} w_{2}-D_{1}^{2} w_{1}-c D_{1} w_{1}-c D_{0} w_{2}+\frac{4 \alpha_{2} V_{A C} V_{D C}}{\left(1-w_{s}\right)^{3}} \cos \left(T_{0} \Omega\right) w_{1}+\frac{\alpha_{2} V_{A C}^{2}}{\left(1-w_{s}\right)^{3}} \cos \left(2 \Omega T_{0}\right) w_{1} \\
+\frac{6 \alpha_{2} V_{E f f D C}}{\left(1-w_{s}\right)^{4}} w_{1} w_{2}+2 \alpha_{1} w_{s}^{\prime \prime}\left(\int_{0}^{1} w_{1}^{\prime} w_{2}^{\prime} \mathrm{d} x\right)+2 \alpha_{1} w_{2}^{\prime \prime}\left(\int_{0}^{1} w_{s}^{\prime} w_{1}^{\prime} \mathrm{d} x\right)+2 \alpha_{1} w_{1}^{\prime \prime}\left(\int_{0}^{1} w_{s}^{\prime} w_{2}^{\prime} \mathrm{d} x\right)+\alpha_{1} w_{1}^{\prime}\left(\int_{0}^{1}\left(w_{1}^{\prime}\right)^{2} \mathrm{~d} x\right)
\end{gathered}
$$

We notice in the equation the participation of both forcing terms in the second and third orders indicating the importance of these terms. Furthermore, the MTS procedure is followed taking into consideration two aspects: First, the self-adjoint mode shapes of the linear part in Eq. (26) have to be solved and verified to obtain the solvability conditions. Second, the particular solution shall have correction terms for the method of reconstitution [312] to for the treatment of the $D_{1}^{2} w_{1}$ term in the third order. Then, the full solution is expressed as

$$
\begin{aligned}
w & =w_{s}+a_{m} \phi_{m}(x) \cos \left(\beta_{m}+\Omega t\right)+\frac{\mu_{1}}{4 \omega_{m}} a_{m} \phi_{m}(x) \sin \left(\Omega t+\beta_{m}\right)+\frac{\bar{F}_{2 V D C}}{2 \omega_{m}^{2}} \phi_{m}(x) \cos (\Omega t) \\
& +\frac{R_{1}}{8 \omega_{m}^{2}} a_{m} a_{n} \phi_{m}(x) \cos \left(\Omega t+\beta_{n}-\beta_{m}\right)+\frac{a_{n}^{2}}{2} \psi_{4}(x)+\frac{a_{m}^{2}}{2} \psi_{3}(x)+a_{n} \phi_{n}(x) \cos \left(2 \Omega t+\beta_{n}\right) \\
& +\frac{\mu_{2}}{4 \omega_{n}} a_{n} \phi_{n}(x) \sin \left(2 \Omega t+\beta_{n}\right)+\frac{\bar{F}_{2 V A C}}{2 \omega_{n}^{2}} \phi_{n}(x) \cos (2 \Omega t)+\frac{R_{2}}{8 \omega_{n}^{2}} a_{m}^{2} \phi_{n}(x) \cos \left(2 \Omega t+2 \beta_{m}\right) \\
& +\frac{a_{n}^{2}}{2} \psi_{2}(x) \cos \left(4 \Omega t+2 \beta_{n}\right)+\frac{a_{m} a_{n}}{2} \psi_{1}(x) \cos \left(3 \Omega t+\beta_{m}+\beta_{n}\right)
\end{aligned}
$$

where $\phi_{i}(x)$ are the mode shapes and $\psi_{i}(x)$ are the particular solutions.

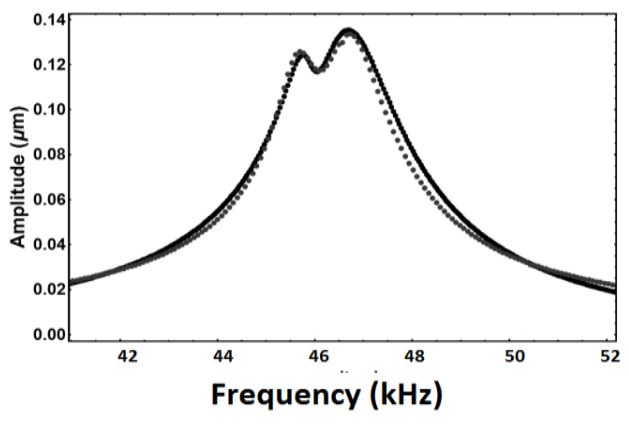

(a)

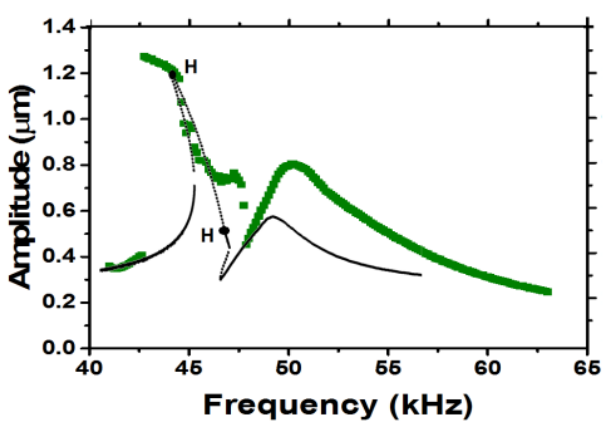

(b)

Fig.17. Frequency response curves for various loading cases. (a) $V_{D C}=40 \quad V_{A C}=0.5$. (b) $V_{D C}=15 \quad V_{A C}=35$. (-) Multiple time scales stable solution. (----) Multiple time scales unstable solution. (•) Solution obtained by solving the system using 4 modes via Galerkin method. ( $\square$ ) Experimental results. H denotes Hopf bifurcation. 
Figure 17 demonstrates some results as compared to numerical (a) and experimental data (b) [307] showing good agreement. One can see that the solution is able to capture the saddle-node jumps associated with the internal resonance interaction and the Hopf bifurcation of the response as noted in Fig. 17b.

One of the shortcoming of the procedure discussed is the fact that there is a need to determine the important terms resulting from the MTS procedure depending on the applied voltage. Another limitation is observed at higher voltage because the procedure presented account only for three terms up to cubic nonlinearity while MEMS, in general, can have higher order nonlinearities.

\section{Conclusions}

MEMS and NEMS possess rich linear and nonlinear dynamical behavior that can be avoided if undesired or exploited for various potential applications. Understanding the dynamical behavior of M/NEMS is crucial for the safe implementation of these structures in different technologies. Exploiting several dynamical approaches, such as the higher order modes, the bifurcation instabilities, and the multimode excitation for sensing application has been reviewed in this paper. Further, the linear and the nonlinear energy transfer among different modes of M/NEMS structures, single and coupled M/NEMS structures, has been reviewed. The focus on linear coupling part was mainly on the veering and mode localization phenomena while the nonlinear coupling was mainly reviewed through internal resonance. We ended up by reviewing different works utilizing the multiple scales technique to model the dynamical behavior as experiencing primary, secondary, and internal resonances. To conclude, MEMS and NEMS resonators and devices are rich in interesting and intriguing dynamical phenomena that can provide valuable opportunities for fundamental and applied research. Understanding the rich and complex dynamical behavior of MEMES and NEMS can open different pathways to implement these systems into wide spectrum of revolutionary applications and technologies.

\section{Acknowledgment:}

This research was support by KAUST.

\section{References}

[1] N. Jaber, S. Ilyas, O. Shekhah, M. Eddaoudi, M.I. Younis, Multimode MEMS Resonator for Simultaneous Sensing of Vapor Concentration and Temperature, IEEE Sensors Journal, 18 (2018) 10145-10153. 
[2] E. Sage, A. Brenac, T. Alava, R. Morel, C. Dupré, M.S. Hanay, M.L. Roukes, L. Duraffourg, C. Masselon, S. Hentz, Neutral particle mass spectrometry with nanomechanical systems, Nature communications, 6 (2015) 6482.

[3] M.S. Hanay, S. Kelber, A. Naik, D. Chi, S. Hentz, E. Bullard, E. Colinet, L. Duraffourg, M. Roukes, Single-protein nanomechanical mass spectrometry in real time, Nature nanotechnology, 7 (2012) 602.

[4] M.A. Al Hafiz, L. Kosuru, A.Z. Hajjaj, M.I. Younis, Highly Tunable Narrow Bandpass MEMS Filter, IEEE Transactions on Electron Devices, 64 (2017) 3392-3398.

[5] S. Ilyas, K. Chappanda, M.I. Younis, Exploiting nonlinearities of micro-machined resonators for filtering applications, Applied Physics Letters, 110 (2017) 253508.

[6] C. Kharrat, E. Colinet, L. Duraffourg, S. Hentz, P. Andreucci, A. Voda, Modal control of mechanically coupled NEMS arrays for tunable RF filters, IEEE transactions on ultrasonics, ferroelectrics, and frequency control, 57 (2010) 1285-1295.

[7] Y. Shim, Z. Wu, M. Rais-Zadeh, A high-performance continuously tunable MEMS bandpass filter at $1 \mathrm{GHz}$, IEEE Transactions on Microwave Theory and Techniques, 60 (2012) 2439-2447.

[8] H. Noh, S.-B. Shim, M. Jung, Z.G. Khim, J. Kim, A mechanical memory with a de modulation of nonlinear resonance, Applied Physics Letters, 97 (2010) 033116.

[9] A. Uranga, J. Verd, E. Marigó, J. Giner, J. Muñóz-Gamarra, N. Barniol, Exploitation of non-linearities in CMOSNEMS electrostatic resonators for mechanical memories, Sensors and Actuators A: Physical, 197 (2013) 88-95.

[10] N. Khovanova, J. Windelen, Minimal energy control of a nanoelectromechanical memory element, Applied Physics Letters, 101 (2012) 024104.

[11] M.A.A. Hafiz, L. Kosuru, M.I. Younis, Microelectromechanical reprogrammable logic device, Nature communications, 7 (2016) 11137.

[12] D.N. Guerra, A.R. Bulsara, W.L. Ditto, S. Sinha, K. Murali, P. Mohanty, A noise-assisted reprogrammable nanomechanical logic gate, Nano letters, 10 (2010) 1168-1171.

[13] I. Mahboob, E. Flurin, K. Nishiguchi, A. Fujiwara, H. Yamaguchi, Interconnect-free parallel logic circuits in a single mechanical resonator, Nature communications, 2 (2011) 198.

[14] I.P. Prikhodko, A.A. Trusov, A.M. Shkel, Compensation of drifts in high-Q MEMS gyroscopes using temperature self-sensing, Sensors and Actuators A: Physical, 201 (2013) 517-524.

[15] J. Fei, J. Zhou, Robust adaptive control of MEMS triaxial gyroscope using fuzzy compensator, IEEE Transactions on Systems, Man, and Cybernetics, Part B (Cybernetics), 42 (2012) 1599-1607.

[16] A. Raman, J. Melcher, R. Tung, Cantilever dynamics in atomic force microscopy, Nano today, 3 (2008) 20-27.

[17] R. Garcia, R. Perez, Dynamic atomic force microscopy methods, Surface science reports, 47 (2002) 197-301.

[18] R. Batra, M. Porfiri, D. Spinello, Review of modeling electrostatically actuated microelectromechanical systems, Smart Materials and Structures, 16 (2007) R23.

[19] J. Tamayo, P.M. Kosaka, J.J. Ruz, Á. San Paulo, M. Calleja, Biosensors based on nanomechanical systems, Chemical Society Reviews, 42 (2013) 1287-1311.

[20] R. Lifshitz, M. Cross, Nonlinear dynamics of nanomechanical and micromechanical resonators, Review of nonlinear dynamics and complexity, 1 (2008) 1-52. 
[21] C. Zhao, M.H. Montaseri, G.S. Wood, S.H. Pu, A.A. Seshia, M. Kraft, A review on coupled MEMS resonators for sensing applications utilizing mode localization, Sensors and Actuators A: Physical, 249 (2016) 93-111.

[22] K. Eom, H.S. Park, D.S. Yoon, T. Kwon, Nanomechanical resonators and their applications in biological/chemical detection: nanomechanics principles, Physics Reports, 503 (2011) 115-163.

[23] W.-C. Chuang, H.-L. Lee, P.-Z. Chang, Y.-C. Hu, Review on the modeling of electrostatic MEMS, Sensors, 10 (2010) 6149-6171.

[24] K. Asadi, J. Yu, H. Cho, Nonlinear couplings and energy transfers in micro-and nano-mechanical resonators: intermodal coupling, internal resonance and synchronization, Philosophical Transactions of the Royal Society A: Mathematical, Physical and Engineering Sciences, 376 (2018) 20170141.

[25] J.F. Rhoads, S.W. Shaw, K.L. Turner, Nonlinear Dynamics and Its Applications in Micro-and Nanoresonators, Journal of Dynamic Systems, Measurement, and Control, 132 (2010) 034001.

[26] R. Karabalin, M. Cross, M. Roukes, Nonlinear dynamics and chaos in two coupled nanomechanical resonators, Physical Review B, 79 (2009) 165309.

[27] I. Mahboob, R. Dupuy, K. Nishiguchi, A. Fujiwara, H. Yamaguchi, Hopf and period-doubling bifurcations in an electromechanical resonator, Applied Physics Letters, 109 (2016) 073101.

[28] D. Czaplewski, D. Antonio, J. Guest, D. Lopez, S. Arroyo, D. Zanette, Enhanced synchronization range from non-linear micromechanical oscillators, in: Solid-State Sensors, Actuators and Microsystems (TRANSDUCERS), 2015 Transducers-2015 18th International Conference on, IEEE, 2015, pp. 2001-2004.

[29] D. Pu, R. Huan, X. Wei, Frequency stability improvement for piezoresistive micromechanical oscillators via synchronization, AIP Advances, 7 (2017) 035204.

[30] D. Pu, X. Wei, L. Xu, Z. Jiang, R. Huan, Synchronization of electrically coupled micromechanical oscillators with a frequency ratio of 3: 1, Applied Physics Letters, 112 (2018) 013503.

[31] D. Antonio, D.H. Zanette, D. López, Frequency stabilization in nonlinear micromechanical oscillators, Nature communications, 3 (2012) 806.

[32] A.Z. Hajjaj, F.K. Alfosail, M.I. Younis, Two-to-one internal resonance of MEMS arch resonators, International Journal of Non-Linear Mechanics, 107 (2018) 64-72.

[33] A. Hajjaj, N. Jaber, M. Hafiz, S. Ilyas, M. Younis, Multiple internal resonances in MEMS arch resonators, Physics Letters A, (2018).

[34] A.Z. Hajjaj, N. Alcheikh, M.I. Younis, The static and dynamic behavior of MEMS arch resonators near veering and the impact of initial shapes, International Journal of Non-Linear Mechanics, 95 (2017) 277-286.

[35] A.Z. Hajjaj, M.A. Hafiz, M.I. Younis, Mode coupling and nonlinear resonances of MEMS arch resonators for bandpass filters, Scientific Reports, 7 (2017) 41820.

[36] V. Sazonova, Y. Yaish, H. Üstünel, D. Roundy, T.A. Arias, P.L. McEuen, A tunable carbon nanotube electromechanical oscillator, Nature, 431 (2004) 284.

[37] L. Ruzziconi, M.I. Younis, S. Lenci, An electrically actuated imperfect microbeam: dynamical integrity for interpreting and predicting the device response, Meccanica, 48 (2013) 1761-1775. 
[38] L. Ruzziconi, M.I. Younis, S. Lenci, Multistability in an electrically actuated carbon nanotube: a dynamical integrity perspective, Nonlinear Dynamics, 74 (2013) 533-549.

[39] L. Ruzziconi, A. Ramini, M. Younis, S. Lenci, Theoretical prediction of experimental jump and pull-in dynamics in a MEMS sensor, Sensors, 14 (2014) 17089-17111.

[40] V. Settimi, G. Rega, Influence of a locally-tailored external feedback control on the overall dynamics of a noncontact AFM model, International Journal of Non-Linear Mechanics, 80 (2016) 144-159.

[41] V. Settimi, O. Gottlieb, G. Rega, Asymptotic analysis of a noncontact AFM microcantilever sensor with external feedback control, Nonlinear Dynamics, 79 (2015) 2675-2698.

[42] A. Shibata, S. Ohishi, H. Yabuno, Passive method for controlling the nonlinear characteristics in a parametrically excited hinged-hinged beam by the addition of a linear spring, Journal of Sound and Vibration, 350 (2015) 111-122.

[43] H.S. Haghighi, A.H. Markazi, Chaos prediction and control in MEMS resonators, Communications in Nonlinear Science and Numerical Simulation, 15 (2010) 3091-3099.

[44] K. Higashino, H. Yabuno, K. Aono, Y. Yamamoto, M. Kuroda, Self-excited vibrational cantilever-type viscometer driven by piezo-actuator, Journal of Vibration and Acoustics, 137 (2015) 061009.

[45] D.I. Caruntu, I. Martinez, Reduced order model of parametric resonance of electrostatically actuated MEMS cantilever resonators, International Journal of Non-Linear Mechanics, 66 (2014) 28-32.

[46] A. Parent, A. Krust, G. Lorenz, T. Piirainen, A novel model order reduction approach for generating efficient nonlinear verilog-a models of mems gyroscopes, in: 2015 IEEE International Symposium on Inertial Sensors and Systems (ISISS) Proceedings, IEEE, 2015, pp. 1-4.

[47] V. Zega, C. Comi, P. Fedeli, A. Frangi, A. Corigliano, P. Minotti, G. Langfelder, L. Falorni, A. Tocchio, A dualmass frequency-modulated (FM) pitch gyroscope: Mechanical design and modelling, in: 2018 IEEE International Symposium on Inertial Sensors and Systems (INERTIAL), IEEE, 2018, pp. 1-4.

[48] G. Longo, L. Alonso-Sarduy, L.M. Rio, A. Bizzini, A. Trampuz, J. Notz, G. Dietler, S. Kasas, Rapid detection of bacterial resistance to antibiotics using AFM cantilevers as nanomechanical sensors, Nature nanotechnology, 8 (2013) 522.

[49] U. Sungkanak, A. Sappat, A. Wisitsoraat, C. Promptmas, A. Tuantranont, Ultrasensitive detection of Vibrio cholerae $\mathrm{O} 1$ using microcantilever-based biosensor with dynamic force microscopy, Biosensors and Bioelectronics, 26 (2010) 784-789.

[50] J. Chaste, A. Eichler, J. Moser, G. Ceballos, R. Rurali, A. Bachtold, A nanomechanical mass sensor with yoctogram resolution, Nature nanotechnology, 7 (2012) 301.

[51] M. Kelleci, H. Aydogmus, L. Aslanbas, S.O. Erbil, M.S. Hanay, Towards microwave imaging of cells, Lab on a Chip, 18 (2018) 463-472.

[52] H. Yamagiwa, S. Sato, T. Fukawa, T. Ikehara, R. Maeda, T. Mihara, M. Kimura, Detection of volatile organic compounds by weight-detectable sensors coated with metal-organic frameworks, Scientific reports, 4 (2014) 6247.

[53] M. Urbiztondo, A. Peralta, I. Pellejero, J. Sesé, M. Pina, I. Dufour, J. Santamaria, Detection of organic vapours with Si cantilevers coated with inorganic (zeolites) or organic (polymer) layers, Sensors and Actuators B: Chemical, 171 (2012) 822-831. 
[54] M. Urbiztondo, I. Pellejero, M. Villarroya, J. Sesé, M.P. Pina, I. Dufour, J. Santamaria, Zeolite-modified cantilevers for the sensing of nitrotoluene vapors, Sensors and Actuators B: Chemical, 137 (2009) 608-616.

[55] Y. Hwang, H. Sohn, A. Phan, O.M. Yaghi, R.N. Candler, Dielectrophoresis-assembled zeolitic imidazolate framework nanoparticle-coupled resonators for highly sensitive and selective gas detection, Nano letters, 13 (2013) 5271-5276.

[56] D.J. Wales, J. Grand, V.P. Ting, R.D. Burke, K.J. Edler, C.R. Bowen, S. Mintova, A.D. Burrows, Gas sensing using porous materials for automotive applications, Chemical Society Reviews, 44 (2015) 4290-4321.

[57] M. Holzinger, A. Le Goff, S. Cosnier, Nanomaterials for biosensing applications: a review, Frontiers in chemistry, 2 (2014) 63.

[58] P.S. Waggoner, H.G. Craighead, Micro-and nanomechanical sensors for environmental, chemical, and biological detection, Lab on a Chip, 7 (2007) 1238-1255.

[59] W.-M. Zhang, H. Yan, Z.-K. Peng, G. Meng, Electrostatic pull-in instability in MEMS/NEMS: A review, Sensors and Actuators A: Physical, 214 (2014) 187-218.

[60] S. Fanget, S. Hentz, P. Puget, J. Arcamone, M. Matheron, E. Colinet, P. Andreucci, L. Duraffourg, E. Myers, M. Roukes, Gas sensors based on gravimetric detection-A review, Sensors and Actuators B: Chemical, 160 (2011) 804821.

[61] R. Abdolvand, B. Bahreyni, J. Lee, F. Nabki, Micromachined resonators: A review, Micromachines, 7 (2016) 160.

[62] A. Ahmed, J. Dennis, M. Md Khir, M. Mohamad Saad, Design and simulation of mass-sensitive gas sensor based on CMOS-MEMS resonator, in: AIP Conference Proceedings, AIP, 2012, pp. 673-676.

[63] I. Bargatin, I. Kozinsky, M. Roukes, Efficient electrothermal actuation of multiple modes of high-frequency nanoelectromechanical resonators, Applied Physics Letters, 90 (2007) 093116.

[64] H. Cho, M.-F. Yu, A.F. Vakakis, L.A. Bergman, D.M. McFarland, Tunable, broadband nonlinear nanomechanical resonator, Nano letters, 10 (2010) 1793-1798.

[65] S. Dohn, R. Sandberg, W. Svendsen, A. Boisen, Enhanced functionality of cantilever based mass sensors using higher modes, Applied Physics Letters, 86 (2005) 233501.

[66] D. Jin, X. Li, J. Liu, G. Zuo, Y. Wang, M. Liu, H. Yu, High-mode resonant piezoresistive cantilever sensors for tens-femtogram resoluble mass sensing in air, Journal of Micromechanics and Microengineering, 16 (2006) 1017.

[67] F. Lochon, I. Dufour, D. Rebiere, An alternative solution to improve sensitivity of resonant microcantilever chemical sensors: comparison between using high-order modes and reducing dimensions, Sensors and Actuators B: Chemical, 108 (2005) 979-985.

[68] S. Olcum, N. Cermak, S.C. Wasserman, S.R. Manalis, High-speed multiple-mode mass-sensing resolves dynamic nanoscale mass distributions, Nature communications, 6 (2015) 7070.

[69] E. Finot, T. Thundat, E. Lesniewska, J.P. Goudonnet, Measuring magnetic susceptibilities of nanogram quantities of materials using microcantilevers, Ultramicroscopy, 86 (2001) 175-180.

[70] T. Ono, M. Esashi, Mass sensing with resonating ultra-thin silicon beams detected by a double-beam laser Doppler vibrometer, Measurement Science and Technology, 15 (2004) 1977. 
[71] J. Teva, G. Abadal, F. Torres, J. Verd, F. Perez-Murano, N. Barniol, A femtogram resolution mass sensor platform, based on SOI electrostatically driven resonant cantilever. Part I: Electromechanical model and parameter extraction, Ultramicroscopy, 106 (2006) 800-807.

[72] N.V. Lavrik, P.G. Datskos, Femtogram mass detection using photothermally actuated nanomechanical resonators, Applied physics letters, 82 (2003) 2697-2699.

[73] I.-B. Baek, S. Byun, B.K. Lee, J.-H. Ryu, Y. Kim, Y.S. Yoon, W.I. Jang, S. Lee, H.Y. Yu, Attogram mass sensing based on silicon microbeam resonators, Scientific Reports, 7 (2017) 46660.

[74] Y.-T. Yang, C. Callegari, X. Feng, K.L. Ekinci, M.L. Roukes, Zeptogram-scale nanomechanical mass sensing, Nano letters, 6 (2006) 583-586.

[75] E. Sage, M. Sansa, S. Fostner, M. Defoort, M. Gély, A.K. Naik, R. Morel, L. Duraffourg, M.L. Roukes, T. Alava, Single-particle mass spectrometry with arrays of frequency-addressed nanomechanical resonators, Nature communications, 9 (2018) 3283.

[76] R. Gao, Y. Huang, X. Wen, J. Zhao, S. Liu, Method to further improve sensitivity for high-order vibration mode mass sensors with stepped cantilevers, IEEE Sensors Journal, 17 (2017) 4405-4411.

[77] M. Okada, H. Nagasaki, A. Tamano, K. Niki, H. Tanigawa, K. Suzuki, Silicon beam resonator utilizing the thirdorder bending mode, Japanese Journal of Applied Physics, 48 (2009) 06FK03.

[78] S. Kuroda, N. Suzuki, H. Tanigawa, K. Suzuki, Variable resonance frequency selection for fishbone-shaped microelectromechanical system resonator based on multi-physics simulation, Japanese Journal of Applied Physics, 52 (2013) 06GL14.

[79] N. Jaber, A. Ramini, A.A. Carreno, M.I. Younis, Higher order modes excitation of electrostatically actuated clamped-clamped microbeams: experimental and analytical investigation, Journal of Micromechanics and Microengineering, 26 (2016) 025008.

[80] R.S. Lakshmanan, R. Mutharasan, Nature of sensitive high-order resonant modes in piezoelectric excited millimeter sized cantilever (PEMC) sensors, Sensors and Actuators A: Physical, 171 (2011) 79-86.

[81] H.C. Nathanson, W.E. Newell, R.A. Wickstrom, J.R. Davis, The resonant gate transistor, IEEE Transactions on Electron Devices, 14 (1967) 117-133.

[82] G.N. Nielson, G. Barbastathis, Dynamic pull-in of parallel-plate and torsional electrostatic MEMS actuators, Journal of microelectromechanical systems, 15 (2006) 811-821.

[83] M.I. Younis, E.M. Abdel-Rahman, A. Nayfeh, A reduced-order model for electrically actuated microbeam-based MEMS, Journal of Microelectromechanical systems, 12 (2003) 672-680.

[84] M.I. Younis, MEMS linear and nonlinear statics and dynamics, Springer Science \& Business Media, 2011.

[85] V. Kumar, Y. Yang, J.W. Boley, G.T.-C. Chiu, J.F. Rhoads, Modeling, analysis, and experimental validation of a bifurcation-based microsensor, Journal of Microelectromechanical Systems, 21 (2012) 549-558.

[86] O.Y. Loh, H.D. Espinosa, Nanoelectromechanical contact switches, Nature nanotechnology, 7 (2012) 283.

[87] M. Khater, K. Vummidi, E. Abdel-Rahman, A. Nayfeh, S. Raman, Dynamic actuation methods for capacitive MEMS shunt switches, Journal of Micromechanics and Microengineering, 21 (2011) 035009. 
[88] A. Ramini, M.I. Younis, Q.T. Su, Low-G Electrostatically Actuated Resonant Switch, in: ASME 2012 International Design Engineering Technical Conferences and Computers and Information in Engineering Conference, American Society of Mechanical Engineers, 2012, pp. 75-85.

[89] R.A. Dias, E. Cretu, R. Wolffenbuttel, L.A. Rocha, Pull-in-based $\mu$ g-resolution accelerometer: Characterization and noise analysis, Sensors and Actuators A: Physical, 172 (2011) 47-53.

[90] M. Khater, M. Al-Ghamdi, S. Park, K. Stewart, E. Abdel-Rahman, A. Penlidis, A. Nayfeh, A. Abdel-Aziz, M. Basha, Binary MEMS gas sensors, Journal of Micromechanics and Microengineering, 24 (2014) 065007.

[91] N. Jaber, S. Ilyas, O. Shekhah, M. Eddaoudi, M.I. Younis, Resonant Gas Sensor and Switch Operating in Air With Metal-Organic Frameworks Coating, Journal of Microelectromechanical Systems, 27 (2018) 156-163.

[92] A. Bouchaala, N. Jaber, O. Shekhah, V. Chernikova, M. Eddaoudi, M.I. Younis, A smart microelectromechanical sensor and switch triggered by gas, Applied Physics Letters, 109 (2016) 013502.

[93] M. Al-Ghamdi, M. Khater, K. Stewart, A. Alneamy, E. Abdel-Rahman, A. Penlidis, Dynamic bifurcation MEMS gas sensors, Journal of Micromechanics and Microengineering, 29 (2018) 015005.

[94] N. Jaber, S. Ilyas, O. Shekhah, M. Eddaoudi, M. Younis, Multimode excitation of a metal organics frameworks coated microbeam for smart gas sensing and actuation, Sensors and Actuators A: Physical, 283 (2018) 254-262.

[95] V.-N. Nguyen, S. Baguet, C.-H. Lamarque, R. Dufour, Bifurcation-based micro-/nanoelectromechanical mass detection, Nonlinear Dynamics, 79 (2015) 647-662.

[96] A. Bouchaala, N. Jaber, O. Yassine, O. Shekhah, V. Chernikova, M. Eddaoudi, M.I. Younis, Nonlinear-based MEMS sensors and active switches for gas detection, Sensors, 16 (2016) 758.

[97] V. Kumar, J.W. Boley, Y. Yang, H. Ekowaluyo, J.K. Miller, G.T.-C. Chiu, J.F. Rhoads, Bifurcation-based mass sensing using piezoelectrically-actuated microcantilevers, Applied Physics Letters, 98 (2011) 153510.

[98] W. Zhang, K.L. Turner, Application of parametric resonance amplification in a single-crystal silicon microoscillator based mass sensor, Sensors and Actuators A: Physical, 122 (2005) 23-30.

[99] W. Zhang, R. Baskaran, K.L. Turner, Effect of cubic nonlinearity on auto-parametrically amplified resonant MEMS mass sensor, Sensors and Actuators A: Physical, 102 (2002) 139-150.

[100] M.I. Younis, F. Alsaleem, Exploration of new concepts for mass detection in electrostatically-actuated structures based on nonlinear phenomena, Journal of computational and nonlinear dynamics, 4 (2009) 021010.

[101] B.N. Johnson, R. Mutharasan, The origin of low-order and high-order impedance-coupled resonant modes in piezoelectric-excited millimeter-sized cantilever (PEMC) sensors: Experiments and finite element models, Sensors and Actuators B: Chemical, 155 (2011) 868-877.

[102] A.R. Kadam, G.P. Nordin, M.A. George, Use of thermally induced higher order modes of a microcantilever for mercury vapor detection, Journal of applied physics, 99 (2006) 094905.

[103] D. Maraldo, R. Mutharasan, Mass-change sensitivity of high-order mode of piezoelectric-excited millimetersized cantilever (PEMC) sensors: theory and experiments, Sensors and Actuators B: Chemical, 143 (2010) 731-739. [104] G. Chawla, S.D. Solares, Mapping of conservative and dissipative interactions in bimodal atomic force microscopy using open-loop and phase-locked-loop control of the higher eigenmode, Applied Physics Letters, 99 (2011) 074103. 
[105] C. Dietz, M. Schulze, A. Voss, C. Riesch, R.W. Stark, Bimodal frequency-modulated atomic force microscopy with small cantilevers, Nanoscale, 7 (2015) 1849-1856.

[106] S. Benaglia, V.G. Gisbert, A.P. Perrino, C.A. Amo, R. Garcia, Fast and high-resolution mapping of elastic properties of biomolecules and polymers with bimodal AFM, Nature protocols, 13 (2018) 2890.

[107] A. Bouchaala, A.H. Nayfeh, N. Jaber, M.I. Younis, Mass and position determination in MEMS mass sensors: a theoretical and an experimental investigation, Journal of Micromechanics and Microengineering, 26 (2016) 105009.

[108] M.I. Younis, Multi-mode excitation of a clamped-clamped microbeam resonator, Nonlinear Dynamics, 80 (2015) 1531-1541.

[109] R. Garcia, R. Proksch, Nanomechanical mapping of soft matter by bimodal force microscopy, European Polymer Journal, 49 (2013) 1897-1906.

[110] S. Patil, N.F. Martinez, J.R. Lozano, R. Garcia, Force microscopy imaging of individual protein molecules with sub-pico Newton force sensitivity, Journal of Molecular Recognition: An Interdisciplinary Journal, 20 (2007) 516523.

[111] T.R. Rodriguez, R. García, Compositional mapping of surfaces in atomic force microscopy by excitation of the second normal mode of the microcantilever, Applied Physics Letters, 84 (2004) 449-451.

[112] R. Ohta, H. Okamoto, H. Yamaguchi, Feedback control of multiple mechanical modes in coupled micromechanical resonators, Applied Physics Letters, 110 (2017) 053106.

[113] H. Okamoto, R. Schilling, H. Schütz, V. Sudhir, D.J. Wilson, H. Yamaguchi, T.J. Kippenberg, A strongly coupled $\Lambda$-type micromechanical system, Applied Physics Letters, 108 (2016) 153105.

[114] G. Verbiest, D. Xu, M. Goldsche, T. Khodkov, S. Barzanjeh, N. Von den Driesch, D. Buca, C. Stampfer, Tunable mechanical coupling between driven microelectromechanical resonators, Applied Physics Letters, 109 (2016) 143507.

[115] Y. Yang, E. Ng, P. Polunin, Y. Chen, S. Strachan, V. Hong, C.H. Ahn, O. Shoshani, S. Shaw, M. Dykman, Experimental investigation on mode coupling of bulk mode silicon MEMS resonators, in: Micro Electro Mechanical Systems (MEMS), 2015 28th IEEE International Conference on, IEEE, 2015, pp. 1008-1011.

[116] X. Wei, T. Zhang, Z. Jiang, J. Ren, R. Huan, Frequency latching in nonlinear micromechanical resonators, Applied Physics Letters, 110 (2017) 143506.

[117] M. Defoort, K. Lulla, C. Blanc, O. Bourgeois, E. Collin, A. Armour, Modal "self-coupling” as a sensitive probe for nanomechanical detection, Applied Physics Letters, 103 (2013) 013104.

[118] J. Güttinger, A. Noury, P. Weber, A.M. Eriksson, C. Lagoin, J. Moser, C. Eichler, A. Wallraff, A. Isacsson, A. Bachtold, Energy-dependent path of dissipation in nanomechanical resonators, Nature nanotechnology, 12 (2017) 631. [119] M. Matheny, L. Villanueva, R. Karabalin, J.E. Sader, M. Roukes, Nonlinear mode-coupling in nanomechanical systems, Nano letters, 13 (2013) 1622-1626.

[120] P. Truitt, J. Hertzberg, E. Altunkaya, K. Schwab, Linear and nonlinear coupling between transverse modes of a nanomechanical resonator, Journal of Applied Physics, 114 (2013) 114307.

[121] C. Chen, D.H. Zanette, D.A. Czaplewski, S. Shaw, D. López, Direct observation of coherent energy transfer in nonlinear micromechanical oscillators, Nature communications, 8 (2017) 15523. 
[122] C. Samanta, P. Yasasvi Gangavarapu, A. Naik, Nonlinear mode coupling and internal resonances in MoS2 nanoelectromechanical system, Applied physics letters, 107 (2015) 173110.

[123] H.M. Ouakad, H.M. Sedighi, M.I. Younis, One-to-one and three-to-one internal resonances in MEMS shallow arches, Journal of Computational and Nonlinear Dynamics, 12 (2017) 051025.

[124] O. Shoshani, S.W. Shaw, M.I. Dykman, Anomalous decay of nanomechanical modes going through nonlinear resonance, Scientific reports, 7 (2017) 18091.

[125] D.H. Zanette, Energy exchange between coupled mechanical oscillators: linear regimes, Journal of Physics Communications, 2 (2018) 095015.

[126] D.H. Zanette, Effects of noise on the internal resonance of a nonlinear oscillator, Scientific reports, 8 (2018) 5976.

[127] D.H. Zanette, Stability of two-mode internal resonance in a nonlinear oscillator, The European Physical Journal B, 91 (2018) 89.

[128] T. Zhang, Z. Jiang, X. Wei, Numerical Investigation of Modal Amplitude Saturation in Micromechanical Cantilever Beam Resonators, in: ASME 2017 International Design Engineering Technical Conferences and Computers and Information in Engineering Conference, American Society of Mechanical Engineers, 2017, pp. V004T009A008-V004T009A008.

[129] G. Labadze, M. Dukalski, Y.M. Blanter, Dynamics of coupled vibration modes in a quantum non-linear mechanical resonator, Physica E: Low-dimensional Systems and Nanostructures, 76 (2016) 181-186.

[130] I. Mahboob, V. Nier, K. Nishiguchi, A. Fujiwara, H. Yamaguchi, Multi-mode parametric coupling in an electromechanical resonator, Applied Physics Letters, 103 (2013) 153105.

[131] D.C. Meier, S. Semancik, B. Button, E. Strelcov, A. Kolmakov, Coupling nanowire chemiresistors with MEMS microhotplate gas sensing platforms, Applied Physics Letters, 91 (2007) 063118.

[132] A.S. Phani, A.A. Seshia, M. Palaniapan, R.T. Howe, J. Yasaitis, Modal coupling in micromechanical vibratory rate gyroscopes, IEEE Sensors Journal, 6 (2006) 1144-1152.

[133] A. Sarrafan, B. Bahreyni, F. Golnaraghi, Analytical Modeling and Experimental Verification of Nonlinear Mode Coupling in a Decoupled Tuning Fork Microresonator, Journal of Microelectromechanical Systems, 27 (2018) 398406.

[134] W.J. Venstra, R. van Leeuwen, H.S. van der Zant, Strongly coupled modes in a weakly driven micromechanical resonator, Applied Physics Letters, 101 (2012) 243111.

[135] D.F. Wang, K. Chatani, T. Ikehara, R. Maeda, Mode localization analysis and characterization in a 5-beam array of coupled nearly identical micromechanical resonators for ultra-sensitive mass detection and analyte identification, Microsystem technologies, 18 (2012) 1923-1929.

[136] W. Xie, H. Lee, S. Lim, Nonlinear dynamic analysis of MEMS switches by nonlinear modal analysis, Nonlinear Dynamics, 31 (2003) 243-256.

[137] T. Yildirim, K. Cho, X. Wu, Y. Lu, Probing the chaotic boundary of a membrane resonator with nanowire arrays, Nanoscale, 9 (2017) 17524-17532. 
[138] T. Zhang, J. Ren, X. Wei, Z. Jiang, R. Huan, Nonlinear coupling of flexural mode and extensional bulk mode in micromechanical resonators, Applied Physics Letters, 109 (2016) 224102.

[139] C. Zhao, G. Sobreviela, M. Pandit, S. Du, X. Zou, A. Seshia, Experimental Observation of Noise Reduction in Weakly Coupled Nonlinear MEMS Resonators, Journal of Microelectromechanical Systems, 26 (2017) 1196-1203.

[140] H. Westra, M. Poot, H. Van Der Zant, W. Venstra, Nonlinear modal interactions in clamped-clamped mechanical resonators, Physical review letters, 105 (2010) 117205.

[141] H. Westra, D. Karabacak, S. Brongersma, M. Crego-Calama, H. Van Der Zant, W. Venstra, Interactions between directly-and parametrically-driven vibration modes in a micromechanical resonator, Physical Review B, 84 (2011) 134305.

[142] R. De Alba, F. Massel, I.R. Storch, T. Abhilash, A. Hui, P.L. McEuen, H.G. Craighead, J.M. Parpia, Tunable phonon-cavity coupling in graphene membranes, Nature nanotechnology, 11 (2016) 741.

[143] A.M. Eriksson, D. Midtvedt, A. Croy, A. Isacsson, Frequency tuning, nonlinearities and mode coupling in circular mechanical graphene resonators, Nanotechnology, 24 (2013) 395702.

[144] R. Liu, L. Wang, Coupling between flexural modes in free vibration of single-walled carbon nanotubes, AIP Advances, 5 (2015) 127110.

[145] W.G. Conley, A. Raman, C.M. Krousgrill, S. Mohammadi, Nonlinear and nonplanar dynamics of suspended nanotube and nanowire resonators, Nano letters, 8 (2008) 1590-1595.

[146] I. Mahboob, K. Nishiguchi, H. Okamoto, H. Yamaguchi, Phonon-cavity electromechanics, Nature Physics, 8 (2012) 387.

[147] N. Perkins, Closed-form vibration analysis of sagged cable/mass suspensions, Ann Arbor, 1050 (1992) 4810942125 .

[148] G. Rega, Theoretical and experimental nonlinear vibrations of sagged elastic cables, in: Nonlinear Dynamic Phenomena in Mechanics, Springer, 2012, pp. 159-210.

[149] A.W. Leissa, On a curve veering aberration, Zeitschrift für angewandte Mathematik und Physik ZAMP, 25 (1974) 99-111.

[150] N. Perkins, C. Mote, Comments on curve veering in eigenvalue problems, Journal of Sound and Vibration, 106 (1986) 451-463.

[151] M. Petyt, C. Fleischer, Free vibration of a curved beam, Journal of Sound and Vibration, 18 (1971) 17-30.

[152] W. Lacarbonara, H.N. Arafat, A.H. Nayfeh, Non-linear interactions in imperfect beams at veering, International Journal of Non-Linear Mechanics, 40 (2005) 987-1003.

[153] A. Erbes, P. Thiruvenkatanathan, J. Woodhouse, A.A. Seshia, Numerical study of the impact of vibration localization on the motional resistance of weakly coupled MEMS resonators, J. Microelectromech. Syst., 24 (2015) 997-1005.

[154] H.M. Ouakad, M.I. Younis, Natural frequencies and mode shapes of slacked carbon nanotube NEMS resonators, in: ASME 2010 International Design Engineering Technical Conferences and Computers and Information in Engineering Conference, American Society of Mechanical Engineers, 2010, pp. 645-652. 
[155] H.M. Ouakad, M.I. Younis, Dynamic response of slacked single-walled carbon nanotube resonators, Nonlinear dynamics, 67 (2012) 1419-1436.

[156] A.H. Ramini, A.Z. Hajjaj, M.I. Younis, Tunable resonators for nonlinear modal interactions, Scientific reports, 6 (2016) 34717.

[157] N. Noori, A. Sarrafan, F. Golnaraghi, B. Bahreyni, Utilization of 2: 1 Internal Resonance in Microsystems, Micromachines, 9 (2018) 448.

[158] J. Charmet, R. Daly, P. Thiruvenkatanathan, J. Woodhouse, A.A. Seshia, Observations of modal interaction in lateral bulk acoustic resonators, Applied Physics Letters, 105 (2014) 013502.

[159] I.B. Flader, C.H. Ahn, Y. Yang, E.J. Ng, V.A. Hong, J. Baek, T.W. Kenny, Tunable quality factor through 1: 1 modal coupling in a disk resonator, in: SENSORS, 2015 IEEE, IEEE, 2015, pp. 1-4.

[160] M.F. Daqaq, E.M. Abdel-Rahman, A.H. Nayfeh, Two-to-one internal resonance in microscanners, Nonlinear Dynamics, 57 (2009) 231.

[161] A. Sarrafan, B. Bahreyni, F. Golnaraghi, Development and characterization of an h-shaped microresonator exhibiting 2: 1 internal resonance, Journal of Microelectromechanical Systems, 26 (2017) 993-1001.

[162] R. Potekin, S. Dharmasena, H. Keum, X. Jiang, J. Lee, S. Kim, L.A. Bergman, A.F. Vakakis, H. Cho, Multifrequency Atomic Force Microscopy based on enhanced internal resonance of an inner-paddled cantilever, Sensors and Actuators A: Physical, 273 (2018) 206-220.

[163] R. Potekin, S. Dharmasena, D.M. McFarland, L.A. Bergman, A.F. Vakakis, H. Cho, Cantilever dynamics in higher-harmonic atomic force microscopy for enhanced material characterization, International Journal of Solids and Structures, 110 (2017) 332-339.

[164] A. Eichler, M. del Álamo Ruiz, J. Plaza, A. Bachtold, Strong coupling between mechanical modes in a nanotube resonator, Physical review letters, 109 (2012) 025503.

[165] C. Lan, W. Qin, W. Deng, Energy harvesting by dynamic unstability and internal resonance for piezoelectric beam, Applied Physics Letters, 107 (2015) 093902.

[166] M.I. Younis, A. Nayfeh, A study of the nonlinear response of a resonant microbeam to an electric actuation, Nonlinear Dynamics, 31 (2003) 91-117.

[167] L. Li, Q. Zhang, W. Wang, J. Han, Nonlinear coupled vibration of electrostatically actuated clamped-clamped microbeams under higher-order modes excitation, Nonlinear Dynamics, 90 (2017) 1593-1606.

[168] A.Z. Hajjaj, N. Alcheikh, M.A.A. Hafiz, S. Ilyas, M.I. Younis, A scalable pressure sensor based on an electrothermally and electrostatically operated resonator, Applied Physics Letters, 111 (2017) 223503.

[169] H.M. Ouakad, M.I. Younis, The dynamic behavior of MEMS arch resonators actuated electrically, International Journal of Non-Linear Mechanics, 45 (2010) 704-713.

[170] M.I. Younis, H.M. Ouakad, F.M. Alsaleem, R. Miles, W. Cui, Nonlinear dynamics of MEMS arches under harmonic electrostatic actuation, Journal of Microelectromechanical Systems, 19 (2010) 647-656.

[171] B.K. Hammad, E.M. Abdel-Rahman, A.H. Nayfeh, Modeling and analysis of electrostatic MEMS filters, Nonlinear Dynamics, 60 (2010) 385-401. 
[172] B.K. Hammad, Natural frequencies and mode shapes of mechanically coupled microbeam resonators with an application to micromechanical filters, Shock and Vibration, 2014 (2014).

[173] S. Pourkamali, F. Ayazi, Electrically coupled MEMS bandpass filters: Part I: With coupling element, Sensors and Actuators A: Physical, 122 (2005) 307-316.

[174] M. Manav, G. Reynen, M. Sharma, E. Cretu, A. Phani, Ultrasensitive resonant MEMS transducers with tuneable coupling, Journal of Micromechanics and Microengineering, 24 (2014) 055005.

[175] S. Pourkamali, F. Ayazi, Electrically coupled MEMS bandpass filters: Part II: Without coupling element, Sensors and Actuators A: Physical, 122 (2005) 317-325.

[176] M. Hajhashemi, A. Amini, B. Bahreyni, A micromechanical bandpass filter with adjustable bandwidth and bidirectional control of centre frequency, Sensors and Actuators A: Physical, 187 (2012) 10-15.

[177] S. Ilyas, K.N. Chappanda, M.A. Al Hafiz, A. Ramini, M.I. Younis, An experimental and theoretical investigation of electrostatically coupled cantilever microbeams, Sensors and Actuators A: Physical, 247 (2016) 368-378.

[178] F.D. Bannon, J.R. Clark, C.-C. Nguyen, High-Q HF microelectromechanical filters, IEEE Journal of solid-state circuits, 35 (2000) 512-526.

[179] C.-Y. Chen, M.-H. Li, C.-S. Li, S.-S. Li, Design and characterization of mechanically coupled CMOS-MEMS filters for channel-select applications, Sensors and Actuators A: Physical, 216 (2014) 394-404.

[180] A.-C. Wong, C.-C. Nguyen, Micromechanical mixer-filters (" mixlers"), Journal of Microelectromechanical Systems, 13 (2004) 100-112.

[181] K. Wang, C.-C. Nguyen, High-order micromechanical electronic filters, in: Micro Electro Mechanical Systems, 1997. MEMS'97, Proceedings, IEEE., Tenth Annual International Workshop on, IEEE, 1997, pp. 25-30.

[182] S. Ilyas, N. Jaber, M.I. Younis, Static and dynamic amplification using strong mechanical coupling, Journal of Microelectromechanical Systems, 25 (2016) 916-921.

[183] R.H. Olsson, C.M. Washburn, J.E. Stevens, M.R. Tuck, C.D. Nordquist, VHF and UHF mechanically coupled aluminum nitride MEMS filters, in: Frequency Control Symposium, 2008 IEEE International, IEEE, 2008, pp. 634639.

[184] H. Zhu, Y. Xu, J.E. Lee, Piezoresistive Readout Mechanically Coupled Lamé Mode SOI Resonator With \$ Q \$ of a Million, Journal of Microelectromechanical Systems, 24 (2015) 771-780.

[185] J.L. Lopez, J. Verd, A. Uranga, J. Giner, G. Murillo, F. Torres, G. Abadal, N. Barniol, A CMOS-MEMS RFtunable bandpass filter based on two high-\$ Q \$22-mhz polysilicon clamped-clamped beam resonators, IEEE Electron Device Letters, 30 (2009) 718-720.

[186] A. Ozgurluk, M. Akgul, C.T.-C. Nguyen, RF channel-select micromechanical disk filters—part I: design, IEEE transactions on ultrasonics, ferroelectrics, and frequency control, 66 (2018) 192-217.

[187] C. Kharrat, E. Colinet, L. Duraffourg, S. Hentz, P. Andreucci, A. Voda, Modal control of mechanically coupled NEMS arrays for tunable RF filters, IEEE transactions on ultrasonics, ferroelectrics, and frequency control, 57 (2010). [188] G. Piazza, Contour-mode aluminum nitride piezoelectric MEMS resonators and filters, in: MEMS-based Circuits and Systems for Wireless Communication, Springer, 2013, pp. 29-54. 
[189] R.H. Olsson, C.M. Washburn, J.E. Stevens, M.R. Tuck, C.D. Nordquist, VHF and UHF mechanically coupled aluminum nitride MEMS filters, in: 2008 IEEE International Frequency Control Symposium, IEEE, 2008, pp. 634639.

[190] C. Zuo, N. Sinha, G. Piazza, Very high frequency channel-select MEMS filters based on self-coupled piezoelectric AlN contour-mode resonators, Sensors and Actuators A: Physical, 160 (2010) 132-140.

[191] G. Piazza, P.J. Stephanou, A.P. Pisano, Single-chip multiple-frequency ALN MEMS filters based on contourmode piezoelectric resonators, Journal of Microelectromechanical Systems, 16 (2007) 319-328.

[192] J. Giner, A. Uranga, J. Muñóz-Gamarra, E. Marigó, N. Barniol, A fully integrated programmable dual-band RF filter based on electrically and mechanically coupled CMOS-MEMS resonators, Journal of Micromechanics and Microengineering, 22 (2012) 055020.

[193] J. Yan, A.A. Seshia, K.L. Phan, J.T. van Beek, Internal electrical and mechanical phase inversion for coupled resonator-array MEMS filters, Sensors and Actuators A: Physical, 158 (2010) 18-29.

[194] S. Ilyas, N. Jaber, M.I. Younis, A Coupled Resonator for Highly Tunable and Amplified Mixer/Filter, IEEE Transactions on Electron Devices, 64 (2017) 2659-2664.

[195] S. Ilyas, N. Jaber, M.I. Younis, A MEMS coupled resonator for frequency filtering in air, Mechatronics, (2018).

[196] S. Ilyas, A. Ramini, A. Arevalo, M.I. Younis, An experimental and theoretical investigation of a micromirror under mixed-frequency excitation, Journal of Microelectromechanical Systems, 24 (2015) 1124-1131.

[197] E. Buks, M.L. Roukes, Electrically tunable collective response in a coupled micromechanical array, Journal of Microelectromechanical Systems, 11 (2002) 802-807.

[198] M.K. Zalalutdinov, J.W. Baldwin, M.H. Marcus, R.B. Reichenbach, J.M. Parpia, B.H. Houston, Twodimensional array of coupled nanomechanical resonators, Applied Physics Letters, 88 (2006) 143504.

[199] M.-H. Li, W.-C. Chen, S.-S. Li, Mechanically coupled CMOS-MEMS free-free beam resonator arrays with enhanced power handling capability, IEEE transactions on ultrasonics, ferroelectrics, and frequency control, 59 (2012) 346-357.

[200] S. Lee, C.-C. Nguyen, Mechanically-coupled micromechanical resonator arrays for improved phase noise, in: Frequency Control Symposium and Exposition, 2004. Proceedings of the 2004 IEEE International, IEEE, 2004, pp. 144-150.

[201] M.U. Demirci, C.T.-C. Nguyen, Mechanically corner-coupled square microresonator array for reduced series motional resistance, Journal of Microelectromechanical Systems, 15 (2006) 1419-1436.

[202] G. Tao, B. Choubey, A simple technique to readout and characterize coupled MEMS resonators, Journal of Microelectromechanical Systems, 25 (2016) 617-625.

[203] G. Tao, H. Zhang, H. Chang, B. Choubey, Inverse Eigenvalue Sensing in Coupled Micro/Nano System, Journal of Microelectromechanical Systems, (2018) 1-10.

[204] E.M. Izhikevich, Computing with oscillators, Neural Networks, (2000).

[205] A. Kumar, P. Mohanty, Autoassociative memory and pattern recognition in micromechanical oscillator network, Scientific reports, 7 (2017) 411. 
[206] F.M. Alsaleem, M.H. Hasan, M.K. Tesfay, A MEMS Nonlinear Dynamic Approach for Neural Computing, Journal of Microelectromechanical Systems, (2018) 1-10.

[207] G. Dion, S. Mejaouri, J. Sylvestre, Reservoir computing with a single delay-coupled non-linear mechanical oscillator, Journal of Applied Physics, 124 (2018) 152132.

[208] C. Pierre, E. Dowell, Localization of vibrations by structural irregularity, Journal of Sound and Vibration, 114 (1987) 549-564.

[209] C. Pierre, Mode localization and eigenvalue loci veering phenomena in disordered structures, Journal of Sound and Vibration, 126 (1988) 485-502.

[210] M. Spletzer, A. Raman, A.Q. Wu, X. Xu, R. Reifenberger, Ultrasensitive mass sensing using mode localization in coupled microcantilevers, Applied Physics Letters, 88 (2006) 254102.

[211] E. Gil-Santos, D. Ramos, A. Jana, M. Calleja, A. Raman, J. Tamayo, Mass sensing based on deterministic and stochastic responses of elastically coupled nanocantilevers, Nano letters, 9 (2009) 4122-4127.

[212] C. Zhao, G. Wood, J. Xie, H. Chang, S.-H. Pu, M. Kraft, Comparative study of different output metrics for a three weakly coupled resonator sensor, in: Solid-State Sensors, Actuators and Microsystems (TRANSDUCERS), 2015 Transducers-2015 18th International Conference on, IEEE, 2015, pp. 2196-2199.

[213] M. Pandit, C. Zhao, G. Sobreviela, A. Mustafazade, A.A. Seshia, Reduction of amplitude ratio dependence on drive level in mode localized resonant MEMS sensors, in: SENSORS, 2017 IEEE, IEEE, 2017, pp. 1-3.

[214] H. Zhang, J. Zhong, J. Yang, W. Yuan, H. Kang, H. Chang, Algebraic summation of eigenstates as a novel output metric to extend the linear sensing range of mode-localized sensors, in: SENSORS, 2017 IEEE, IEEE, 2017, pp. 1-3.

[215] H. Zhang, J. Yang, W. Yuan, H. Chang, Linear sensing for mode-localized sensors, Sensors and Actuators A: Physical, 277 (2018) 35-42.

[216] H. Zhang, H. Chang, W. Yuan, Characterization of forced localization of disordered weakly coupled micromechanical resonators, Microsystems \& Nanoengineering, 3 (2017) 17023.

[217] C. Zhao, M. Pandit, B. Sun, G. Sobreviela, X. Zou, A. Seshia, A closed-Loop readout configuration for modeLocalized resonant MEMS sensors, Journal of Microelectromechanical Systems, 26 (2017) 501-503.

[218] H. Kang, J. Yang, H. Chang, A Closed-Loop Accelerometer Based on Three Degree-of-Freedom Weakly Coupled Resonator With Self-Elimination of Feedthrough Signal, IEEE Sensors Journal, 18 (2018) 3960-3967.

[219] P. Thiruvenkatanathan, J. Yan, A.A. Seshia, Common mode rejection in electrically coupled MEMS resonators

utilizing mode localization for sensor applications, in: Frequency Control Symposium, 2009 Joint with the 22nd European Frequency and Time Forum. IEEE International, IEEE, 2009, pp. 358-363.

[220] H. Zhang, J. Zhong, W. Yuan, J. Yang, H. Chang, Ambient pressure drift rejection of mode-localized resonant sensors, in: Micro Electro Mechanical Systems (MEMS), 2017 IEEE 30th International Conference on, IEEE, 2017, pp. 1095-1098.

[221] J. Zhong, J. Yang, H. Chang, The temperature drift suppression of mode-localized resonant sensors, in: Micro Electro Mechanical Systems (MEMS), 2018 IEEE, IEEE, 2018, pp. 467-470. 
[222] C. Zhao, M. Pandit, G. Sobreviela, A. Mustafazade, S. Du, X. Zou, A. Seshia, On the noise optimization of resonant MEMS sensors utilizing vibration mode localization, Applied Physics Letters, 112 (2018) 194103.

[223] M. Pandit, C. Zhao, A. Mustafazade, G. Sobreviela, A.A. Seshia, Nonlinear cancellation in weakly coupled MEMS resonators, in: Frequency and Time Forum and IEEE International Frequency Control Symposium (EFTF/IFC), 2017 Joint Conference of the European, IEEE, 2017, pp. 16-19.

[224] Y. Wang, C. Zhao, C. Wang, D. Cerica, M. Baijot, Q. Xiao, S. Stoukatch, M. Kraft, A mass sensor based on 3 DOF mode localized coupled resonator under atmospheric pressure, Sensors and Actuators A: Physical, (2018).

[225] G. Tao, B. Choubey, Variability Induced Sensitivity Degradation in Coupled Nano/Micro Resonant Sensors, IEEE Transactions on Nanotechnology, (2018).

[226] M. Spletzer, A. Raman, H. Sumali, J.P. Sullivan, Highly sensitive mass detection and identification using vibration localization in coupled microcantilever arrays, Applied Physics Letters, 92 (2008) 114102.

[227] C. Zhao, G.S. Wood, J. Xie, H. Chang, S.H. Pu, M. Kraft, A force sensor based on three weakly coupled resonators with ultrahigh sensitivity, Sensors and Actuators A: Physical, 232 (2015) 151-162.

[228] P. Thiruvenkatanathan, J. Yan, A.A. Seshia, Ultrasensitive mode-localized micromechanical electrometer, in: Frequency Control Symposium (FCS), 2010 IEEE International, IEEE, 2010, pp. 91-96.

[229] H. Zhang, J. Huang, W. Yuan, H. Chang, A high-sensitivity micromechanical electrometer based on mode localization of two degree-of-freedom weakly coupled resonators, Journal of Microelectromechanical Systems, 25 (2016) 937-946.

[230] J. Yang, J. Zhong, H. Chang, A closed-loop mode-localized accelerometer, Journal of Microelectromechanical Systems, 27 (2018) 210-217.

[231] H. Zhang, B. Li, W. Yuan, M. Kraft, H. Chang, An acceleration sensing method based on the mode localization of weakly coupled resonators, Journal of microelectromechanical systems, 25 (2016) 286-296.

[232] M. Pandit, C. Zhao, G. Sobreviela, A. Mustafazade, X. Zou, A.A. Seshia, A mode-localized MEMS accelerometer with $7 \mu \mathrm{g}$ bias stability, in: Micro Electro Mechanical Systems (MEMS), 2018 IEEE, IEEE, 2018, pp. 968-971.

[233] M. Mojahedi, M. Moghimi Zand, M.T. Ahmadian, Static pull-in analysis of electrostatically actuated microbeams using homotopy perturbation method, Applied Mathematical Modelling, 34 (2010) 1032-1041.

[234] A. Rafiq, M.Y. Malik, T. Abbasi, Solution of nonlinear pull-in behavior in electrostatic micro-actuators by using He's homotopy perturbation method, Computers \& Mathematics with Applications, 59 (2010) 2723-2733.

[235] A.R. Askari, M. Tahani, Stability analysis of electrostatically actuated nano/micro-beams under the effect of van der Waals force, a semi-analytical approach, Communications in Nonlinear Science and Numerical Simulation, 34 (2016) 130-141.

[236] J.R. Beckham, J.A. Pelesko, An electrostatic-elastic membrane system with an external pressure, Mathematical and Computer Modelling, 54 (2011) 2686-2708.

[237] S.M. Lin, M.J. Teng, The analysis and model formulation of a coupled micro-probe and elastic thin plate subjected to electrostatic force, International Journal of Mechanical Sciences, 103 (2015) 224-234. 
[238] A.E. Lindsay, Regularized model of post-touchdown configurations in electrostatic MEMS: bistability analysis, Journal of Engineering Mathematics, 99 (2016) 65-77.

[239] W.D. Yang, F.P. Yang, X. Wang, Dynamic instability and bifurcation of electrically actuated circular nanoplate considering surface behavior and small scale effect, International Journal of Mechanical Sciences, 126 (2017) 12-23. [240] S.M. Shahruz, Suppression of vibration localization in non-axisymmetric periodic structures, Journal of Engineering Mathematics, 62 (2008) 51-65.

[241] S. Krylov, S. Seretensky, Higher order correction of electrostatic pressure and its influence on the pull-in behavior of microstructures, Journal of Micromechanics and Microengineering, 16 (2006) 1382-1396.

[242] M.I. Younis, A.H. Nayfeh, Simulation of Squeeze-Film Damping of Microplates Actuated by Large Electrostatic Load, Journal of Computational and Nonlinear Dynamics, 2 (2007) 232-241.

[243] Y.B. Yi, A. Rahafrooz, S. Pourkamali, Modeling and testing of the collective effects of thermoelastic and fluid damping on silicon MEMS resonators, Journal of Micro-Nanolithography Mems and Moems, 8 (2009).

[244] A.H. Nayfeh, M.I. Younis, Modeling and simulations of thermoelastic damping in microplates, Journal of Micromechanics and Microengineering, 14 (2004) 1711-1717.

[245] M. Zamanian, S.E. Khadem, Analysis of thermoelastic damping in microresonators by considering the stretching effect, International Journal of Mechanical Sciences, 52 (2010) 1366-1375.

[246] S. Salajeghe, S.E. Khadem, M. Rasekh, Nonlinear analysis of thermoelastic damping in axisymmetric vibration of micro circular thin-plate resonators, Applied Mathematical Modelling, 36 (2012) 5991-6000.

[247] Y.B. Yi, Geometric effects on thermoelastic damping in MEMS resonators, Journal of Sound and Vibration, 309 (2008) 588-599.

[248] T. Emig, A. Hanke, R. Golestanian, M. Kardar, Normal and lateral Casimir forces between deformed plates, Physical Review A, 67 (2003).

[249] X.L. Jia, J. Yang, S. Kitipornchai, C.W. Lim, Resonance frequency response of geometrically nonlinear microswitches under electrical actuation, Journal of Sound and Vibration, 331 (2012) 3397-3411.

[250] B.J. Gallacher, J.S. Burdess, K.M. Harish, A control scheme for a MEMS electrostatic resonant gyroscope excited using combined parametric excitation and harmonic forcing, Journal of Micromechanics and Microengineering, 16 (2006) 320-331.

[251] L. Cao, S.C. Fan, Z.S. Guo, L. Xu, A method to simulate the vibrating characters of the resonator for resonant MEMS gyroscope, Microsystem Technologies-Micro-and Nanosystems-Information Storage and Processing Systems, 22 (2016) 2315-2327.

[252] S. Redkar, Reduced-Order Modeling of Parametrically Excited Micro-Electro-Mechanical Systems (MEMS), Advances in Mechanical Engineering, (2010).

[253] A.M. Elshurafa, K. Khirallah, H.H. Tawfik, A. Emira, A. Aziz, S.M. Sedky, Nonlinear Dynamics of Spring Softening and Hardening in Folded-MEMS Comb Drive Resonators, Journal of Microelectromechanical Systems, 20 (2011) 943-958.

[254] W. Zhang, R. Baskaran, K.L. Turner, Effect of cubic nonlinearity on auto-parametrically amplified resonant MEMS mass sensor, Sensors and Actuators A: Physical, 102 (2002) 139-150. 
[255] C.Z. Guo, G.K. Fedder, Behavioral Modeling of a CMOS-MEMS Nonlinear Parametric Resonator, Journal of Microelectromechanical Systems, 22 (2013) 1447-1457.

[256] Q. Li, S. Fan, Z. Tang, W. Xing, Non-linear dynamics of an electrothermally excited resonant pressure sensor, Sensors and Actuators A: Physical, 188 (2012) 19-28.

[257] F.R. Jeffrey, W.S. Steven, L.T. Kimberly, The nonlinear response of resonant microbeam systems with purelyparametric electrostatic actuation, Journal of Micromechanics and Microengineering, 16 (2006) 890.

[258] W. Zhang, G. Meng, Nonlinear Dynamic Analysis of Electrostatically Actuated Resonant MEMS Sensors Under Parametric Excitation, IEEE Sensors Journal, 7 (2007) 370-380.

[259] B.E. DeMartini, J.F. Rhoads, K.L. Turner, S.W. Shaw, J. Moehlis, Linear and Nonlinear Tuning of Parametrically Excited MEMS Oscillators, Journal of Microelectromechanical Systems, 16 (2007) 310-318.

[260] J.F. Rhoads, V. Kumar, S.W. Shaw, K.L. Turner, The non-linear dynamics of electromagnetically actuated microbeam resonators with purely parametric excitations, International Journal of Non-Linear Mechanics, 55 (2013) 79-89.

[261] L. Xu, X. Jia, Electromechanical coupled nonlinear dynamics for microbeams, Archive of Applied Mechanics, 77 (2007) 485-502.

[262] R. Jazar, M. Mahinfalah, N. Mahmoudian, M. Rastgaar, Effects of nonlinearities on the steady state dynamic behavior of electric actuated microcantilever-based resonators, Journal of Vibration and Control, 15 (2009) 12831306.

[263] N. Kacem, S. Hentz, D. Pinto, B. Reig, V. Nguyen, Nonlinear dynamics of nanomechanical beam resonators: improving the performance of NEMS-based sensors, Nanotechnology, 20 (2009) 275501.

[264] P. Kim, S. Bae, J. Seok, Resonant behaviors of a nonlinear cantilever beam with tip mass subject to an axial force and electrostatic excitation, International Journal of Mechanical Sciences, 64 (2012) 232-257.

[265] N. Kacem, S. Baguet, S. Hentz, R. Dufour, Computational and quasi-analytical models for non-linear vibrations of resonant MEMS and NEMS sensors, International Journal of Non-Linear Mechanics, 46 (2011) 532-542.

[266] G. Sarı, M. Pakdemirli, Vibrations of a slightly curved microbeam resting on an elastic foundation with nonideal boundary conditions, Mathematical Problems in Engineering, 2013 (2013).

[267] S. Shao, K. Masri, M.I. Younis, The effect of time-delayed feedback controller on an electrically actuated resonator, Nonlinear Dynamics, 74 (2013) 257-270.

[268] C. Feng, L. Yu, W. Zhang, Dynamic analysis of a dielectric elastomer-based microbeam resonator with large vibration amplitude, International Journal of Non-Linear Mechanics, 65 (2014) 63-68.

[269] J. Peng, L. Yang, G. Luo, J. Yang, Nonlinear electro-dynamic analysis of micro-actuators: Effect of material nonlinearity, Applied Mathematical Modelling, 38 (2014) 2781-2790.

[270] Y.-Z. Wang, F.-M. Li, Nonlinear primary resonance of nano beam with axial initial load by nonlocal continuum theory, International Journal of Non-Linear Mechanics, 61 (2014) 74-79.

[271] J. Han, Q. Zhang, W. Wang, Static bifurcation and primary resonance analysis of a MEMS resonator actuated by two symmetrical electrodes, Nonlinear Dynamics, 80 (2015) 1585-1599. 
[272] A. Bouchaala, A.H. Nayfeh, M.I. Younis, Frequency shifts of micro and nano cantilever beam resonators due to added masses, Journal of Dynamic Systems, Measurement, and Control, 138 (2016) 091002.

[273] A. Bouchaala, A.H. Nayfeh, M.I. Younis, Analytical study of the frequency shifts of micro and nano clampedclamped beam resonators due to an added mass, Meccanica, 52 (2017) 333-348.

[274] L. Li, Q. Zhang, W. Wang, J. Han, Dynamic analysis and design of electrically actuated viscoelastic microbeams considering the scale effect, International Journal of Non-Linear Mechanics, 90 (2017) 21-31.

[275] A. Sabater, J. Rhoads, Parametric system identification of resonant micro/nanosystems operating in a nonlinear response regime, Mechanical Systems and Signal Processing, 84 (2017) 241-264.

[276] M. Ghommem, A. Abdelkefi, Nonlinear reduced-order modeling and effectiveness of electrically-actuated microbeams for bio-mass sensing applications, International Journal of Mechanics and Materials in Design, 1-19.

[277] J. Han, G. Jin, Q. Zhang, W. Wang, B. Li, H. Qi, J. Feng, Dynamic evolution of a primary resonance MEMS resonator under prebuckling pattern, Nonlinear Dynamics, (2018) 1-22.

[278] J. Han, Q. Zhang, W. Wang, G. Jin, B. Li, Stability and perturbation analysis of a one-degree-of-freedom doubly clamped microresonator with delayed velocity feedback control, Journal of Vibration and Control, 24 (2018) 34543470.

[279] Z. Saadatnia, H. Askari, E. Esmailzadeh, Multi-frequency excitation of microbeams supported by Winkler and Pasternak foundations, Journal of Vibration and Control, 24 (2018) 2894-2911.

[280] M.F. Daqaq, E.M. Abdel-Rahman, A.H. Nayfeh, Towards a stable low-voltage torsional microscanner, Microsystem Technologies, 14 (2008) 725-737.

[281] L. Laurent, J.J. Yon, J.S. Moulet, P. Imperinetti, L. Duraffourg, Compensation of nonlinear hardening effect in a nanoelectromechanical torsional resonator, Sensors and Actuators A: Physical, 263 (2017) 326-331.

[282] M. Pandey, R.H. Rand, A.T. Zehnder, Frequency locking in a forced Mathieu-van der Pol-Duffing system, Nonlinear Dynamics, 54 (2008) 3-12.

[283] A. Elnaggar, A. El-Bassiouny, G. Mosa, Harmonic and sub-harmonic resonance of MEMS subjected to a weakly non-linear parametric and external excitations, International Journal of Applied Mathematics Research, 2 (2013) 252.

[284] S. Naik, T. Hikihara, H. Vu, A. Palacios, V. In, P. Longhini, Local bifurcations of synchronization in selfexcited and forced unidirectionally coupled micromechanical resonators, Journal of Sound and Vibration, 331 (2012) 1127-1142.

[285] H.M. Ouakad, A.H. Nayfeh, S. Choura, F. Najar, Nonlinear feedback controller of a microbeam resonator, Journal of Vibration and Control, 21 (2015) 1680-1697.

[286] S.N. Mahmoodi, M.F. Daqaq, N. Jalili, On the nonlinear-flexural response of piezoelectrically driven microcantilever sensors, Sensors and Actuators A: Physical, 153 (2009) 171-179.

[287] S. Azizi, M.R. Ghazavi, G. Rezazadeh, I. Ahmadian, C. Cetinkaya, Tuning the primary resonances of a micro resonator, using piezoelectric actuation, Nonlinear Dynamics, 76 (2014) 839-852.

[288] S. Mehrdad Pourkiaee, S.E. Khadem, M. Shahgholi, Nonlinear vibration and stability analysis of an electrically actuated piezoelectric nanobeam considering surface effects and intermolecular interactions, Journal of Vibration and Control, 23 (2017) 1873-1889. 
[289] F. Tajaddodianfar, H.N. Pishkenari, M.R.H. Yazdi, E.M. Miandoab, On the dynamics of bistable micro/nano resonators: analytical solution and nonlinear behavior, Communications in Nonlinear Science and Numerical Simulation, 20 (2015) 1078-1089.

[290] D.I. Caruntu, L. Luo, Frequency response of primary resonance of electrostatically actuated CNT cantilevers, Nonlinear Dynamics, 78 (2014) 1827-1837.

[291] H.M. Ouakad, A.M. Alofi, A.H. Nayfeh, Dynamic Analysis of Multilayers Based MEMS Resonators, Mathematical Problems in Engineering, (2017).

[292] I. Kirrou, M. Belhaq, Effect of contact stiffness modulation in contact-mode AFM under subharmonic excitation, Communications in Nonlinear Science and Numerical Simulation, 18 (2013) 2916-2925.

[293] E.M. Abdel-Rahman, A.H. Nayfeh, Secondary resonances of electrically actuated resonant microsensors, Journal of Micromechanics and Microengineering, 13 (2003) 491.

[294] N. Kacem, S. Baguet, S. Hentz, R. Dufour, Pull-In Retarding in Nonlinear Nanoelectromechanical Resonators Under Superharmonic Excitation, Journal of Computational and Nonlinear Dynamics, 7 (2012) 021011-021011021018 .

[295] S. Azizi, M.T. Chorsi, F. Bakhtiari-Nejad, On the secondary resonance of a MEMS resonator: A conceptual study based on shooting and perturbation methods, International Journal of Non-Linear Mechanics, 82 (2016) 59-68. [296] A. Elnaggar, A. El-Bassiouny, K. Khalil, A. Omran, Periodic solutions of a modified Duffing equation subjected to a bi-harmonic parametric and external excitations, British Journal of Mathematics and Computer Science, 16 (2016) 1-12.

[297] M.e.S. Sari, Superharmonic resonance analysis of nonlocal nano beam subjected to axial thermal and magnetic forces and resting on a nonlinear elastic foundation, Microsystem Technologies, 23 (2017) 3319-3330.

[298] B.K. Hammad, A.H. Nayfeh, E.M. Abdel-Rahman, On the Use of the Subharmonic Resonance as a Method for Filtration, Journal of Computational and Nonlinear Dynamics, 6 (2011).

[299] S.F. Asokanthan, S.T. Ariaratnam, J.Y. Cho, T.F. Wang, MEMS vibratory angular rate sensors: Stability considerations for design, Structural Control \& Health Monitoring, 13 (2006) 76-90.

[300] S. Ilyas, F. Alfosail, M. Younis, On the Response of MEMS Resonators under Generic Electrostatic Loadings: Theoretical Analysis, Nonlinear Dynamics, (Submitted).

[301] A.-E.M. Elnaggar, A.F. El-Bassiouny, G.A. Mosa, Perturbation analysis of an electrostatic Micro-ElectroMechanical System (MEMS) subjected to external and non-linear parametric excitations, International Journal of Basic and Applied Sciences, 3 (2014) 209.

[302] D. Younesian, M. Sadri, E. Esmailzadeh, Primary and secondary resonance analyses of clamped-clamped microbeams, Nonlinear Dynamics, 76 (2014) 1867-1884.

[303] S. Ilyas, F. Alfosail, M. Younis, On the Application of the Multiple Scales Method on Electrostatically Actuated Resonators, Journal of Computational and Nonlinear Dynamics, (Submitted).

[304] S. Ilyas, F.K. Alfosail, M.I. Younis, On the Application of the Multiple Scales Method on Electrostatically Actuated Resonators, Journal of Computational and Nonlinear Dynamics, 14 (2019) 041006. 
[305] S. Ilyas, F.K. Alfosail, M.L.F. Bellaredj, M.I. Younis, On the response of MEMS resonators under generic electrostatic loadings: experiments and applications, Nonlinear Dynamics, (2018).

[306] M.I. Younis, A.H. Nayfeh, A Study of the Nonlinear Response of a Resonant Microbeam to an Electric Actuation, Nonlinear Dyn., 31 (2003) 91-117.

[307] F.K. Alfosail, A.Z. Hajjaj, M.I. Younis, Theoretical and Experimental Investigation of Two-to-One Internal Resonance in MEMS Arch Resonators, Journal of Computational and Nonlinear Dynamics, 14 (2018) 011001011001-011015.

[308] M.H. Ghayesh, H. Farokhi, Internal energy transfer in dynamical behaviour of Timoshenko microarches, Mathematics and Computers in Simulation, 112 (2015) 28-39.

[309] H.M. Ouakad, H.M. Sedighi, M.I. Younis, One-to-One and Three-to-One Internal Resonances in MEMS Shallow Arches, Journal of Computational and Nonlinear Dynamics, 12 (2017) 051025-051025-051011.

[310] A. Vyas, D. Peroulis, A.K. Bajaj, Dynamics of a nonlinear microresonator based on resonantly interacting flexural-torsional modes, Nonlinear Dynamics, 54 (2008) 31-52.

[311] S.M. Pourkiaee, S.E. Khadem, M. Shahgholi, S. Bab, Nonlinear modal interactions and bifurcations of a piezoelectric nanoresonator with three-to-one internal resonances incorporating surface effects and van der Waals dissipation forces, Nonlinear Dynamics, 88 (2017) 1785-1816.

[312] A.H. Nayfeh, Resolving controversies in the application of the method of multiple scales and the generalized method of averaging, Nonlinear Dynamics, 40 (2005) 61-102. 